\title{
Ground state correlations in a trapped quasi one-dimensional Bose Gas
}

\author{
R. Walser* \\ Abteilung für Quantenphysik, Universität Ulm, D-89069, Ulm, Germany
}

(Dated: 11th January 2019)

\begin{abstract}
We review the basic concepts of a non-equilibrium kinetic theory of a trapped bosonic gas. By extending the successful mean-field concept of the Gross-Pitaevskii equation with the effects of non-local, two particle quantum correlations, one obtains a renormalized binary interaction and allows for the dynamic establishment of non-classical many-particle quantum correlations. These concepts are illustrated by self-consistent numerical calculations of the first and second order ground state quantum correlations of a harmonically trapped, quasi one-dimensional bosonic gas. We do find a strong suppression of the density fluctuations or, in other words, an enhanced number squeezing with decreasing particle density.

PACS numbers: 03.75.Fi, 05.30.Jp, 67.40.Db, 05.70.Ln
\end{abstract}

\section{INTRODUCTION}

Already the state of a single quantum mechanical particle can exhibit an amazingly complexity. Without the restriction to one space dimension or by exploiting additional symmetries, it is virtually impossible even to visualize the information that is encoded in a single particle wave function. Even more so, this situation gets quickly out-off hand by adding more interacting particles to the system. While the new field of quantum information science is exactly trying to accomplish this task of describing and preparing many-particle entanglement, it remains a formidable challenge. In contrast, many-particle physics is blessed with serendipity as the astronomic growth in the dimensionality of the state space leads to a great reduction of complexity. No macroscopic physical phenomenon does rely on a miniscule detail of a particular quantum state as long as the whole ensemble exhibits a certain macroscopic characteristic.

The past decade of research in degenerate atomic gases has produced an amazing wealth of condensed many-particle phenomena [1-4]: Bose-Einstein condensation [5-7], the creation of vortices [8, 9], Abrikosov lattices [10], the Mott phase transition [11-13], the creation of molecular condensates [14, 15], the BCS-BEC crossover [16, 17] and the one-dimensional Tonks-Girardeau gas [18, 19], most of which have been seen or, at least, were predicted in other, traditional fields of low temperature physics. However, observing the original BEC phase transition of a dilute atomic gas in situ and real space is still a delightful lesson in fundamental physics, as one can be an eye witness of the establishment of off-diagonal long range order (ODLRO) [20, 21] and get an impression of role that is carried by quantum fluctuations .

The art of performing successful many-particle calculations consists of picking the right approximation scheme that matches the experimental system on one hand and that is theoretically tractable on the other hand. In the context of weakly correlated dilute Bose gases, the Gross-Pitaevskii (GP) meanfield picture has been a tremendously rewarding concept and the extension to incorporate quantum fluctuations dynami-

*Electronic address: Reinhold.Walser@physik.uni-ulm.de cally is in principle straight forward, although it involves in detail some intricate calculations [22-29]. The description of the temporal relaxation $[30,31]$, the buildup of spatial correlation functions $[32,33]$ and the squeezing of atomic number density fluctuations [34] follow, consequently.

However, it has been recognized very early on that the spatial dimensionality of a system is of utmost importance to its physical behavior. In particular, it has been proven that the reduction of the available phase space volume leads to enhanced fluctuations and the absence of ODLRO in one and two dimensions [35, 36]. During the last years, this fascinating observation has received much attention [37-40] as trapped, inhomogeneous systems violate the translational invariance as required in $[35,36]$. This different physical response should also be reflected by tuning the trap geometry dynamically from $3 \mathrm{~d} \rightarrow 2 \mathrm{~d} \rightarrow$ quasi $1 \mathrm{~d} \rightarrow 1 \mathrm{~d}[18,19,41-43]$. The extended mean-field theory that will be presented in the following, is well suited to describe the cross-over physics with the exception of the strongly interacting Tonks-Girardeau regime.

We have arranged this article according to the following outline: In Sec. II, we will give a basic review of the premises and concepts of non-equilibrium kinetic theory. In particular, we discuss the physical meaning of the relevant master variables that are used. We discuss the derivation and approximations that lead to the the basic self-consistent time-dependent Hartree-Fock-Bogoliubov (HFB) equations of motion in the absence of collision. A short review on the mathematical properties of "Bogoliubov-like" symplectic self energy operators is given, taking special care of the presence or absence of a zero energy mode. Sec. III is devoted to an application of the general formulation to a quasi one-dimensional trapped bosonic gas. To calculate specific numbers, we are assuming in here the typical data of a ${ }^{87} \mathrm{Rb}$ experiment. In particular, we calculate for the zero-temperature ground state of a gas: the mean-amplitude, the quantum depletion, the pairing field, as well as the first and second order correlation functions for a full range of particle numbers $N=\left(10^{0}, \ldots, 10^{5}\right)$. In Sec. IV, we do draw conclusion and give an outlook to the work in progress. Finally, five short appendices compile some technical methods or basic statements that were used in the article. 


\section{COLLISIONLESS KINETIC EQUATIONS}

\section{A. Quantum dynamics}

The kinetic evolution of a trapped atomic gas is described very well by a dilute gas Hamiltonian [1]. In the limit of strongly rarefied atomic gases, it consists primarily of the single particle energy of atoms in a harmonic trap potential $V_{\text {ho }}$ and the mutual interaction energy amongst all pairs of atoms which is mediated through a short-range inter-atomic potential $V_{\text {bin }}$. Strong collisions between atomic triples are very unlikely events in this dilute gas limit and can be disregarded, consequently. Thus, one finds for the Hamilton operator

$$
\begin{array}{r}
\hat{H}=\int d^{3} x \hat{a}_{\mathbf{x}}^{\dagger}\left(-\frac{\hbar^{2}}{2 m} \Delta+V_{\text {ho }}(\mathbf{x})-\mu\right) \hat{a}_{\mathbf{x}} \\
+\frac{1}{2} \int d^{6} x y \hat{a}_{\mathbf{x}}^{\dagger} \hat{a}_{\mathbf{y}}^{\dagger} V_{\text {bin }}(\mathbf{x}-\mathbf{y}) \hat{a}_{\mathbf{y}} \hat{a}_{\mathbf{x}}
\end{array}
$$

where $m$ denotes the atomic mass and $\mu$ is a conveniently chosen zero-energy reference that will be identified later with the chemical potential.

In the language of second quantization, the action of a field operator $\hat{a}_{\mathbf{x}}$ or $\hat{a}_{\mathbf{x}}^{\dagger}$ on a state in Fock space represents the removal or creation of another unstructured particle at the spatial position $\mathbf{x}$. The bosonic nature of the indistinguishable particles is reflected by the commutation relation of the fields as

$$
\left[\hat{a}_{\mathbf{x}}, \hat{a}_{\mathbf{y}}^{\dagger}\right]=\delta(\mathbf{x}-\mathbf{y})
$$

Due to the symmetry $\left[\hat{a}_{\mathbf{x}}, \hat{a}_{\mathbf{y}}\right]=0$ of the bosonic particles under coordinate exchange, only even parity contribution of the interaction potential $V_{\text {bin }}(\mathbf{x})=V_{\text {bin }}(-\mathbf{x})$ contribute to the kinematic evolution.

In principle, all dynamic and static aspects of the evolution of observables $\hat{\mathcal{O}}$ can be obtained from the solution of Heisenberg's equation $d \hat{\mathcal{O}} / d t=i[\hat{H}, \hat{\mathcal{O}}] / \hbar$ and the knowledge of the initial state of the system, which is represented by the many-body density matrix $\rho$. As all observables are formed by the elementary quantum fields $\hat{a}$, it is only necessary to consider the Heisenberg equation

$$
i \hbar \frac{d}{d t} \hat{a}_{\mathbf{x}}(t)=\mathcal{H}(\mathbf{x}) \hat{a}_{\mathbf{x}}+\int d^{3} y V_{\mathrm{bin}}(\mathbf{x}-\mathbf{y}) \hat{a}_{\mathbf{y}}^{\dagger} \hat{a}_{\mathbf{y}} \hat{a}_{\mathbf{x}}
$$

For convenience and later reference, we have introduced in here the Hamilton operator of a single trapped atom

$$
\mathcal{H}(\mathbf{x})=-\frac{\hbar^{2}}{2 m} \Delta+V_{\mathrm{ho}}(\mathbf{x})-\mu
$$

In principle, any complete state representation can be used to perform further calculations. However, it is intuitively clear that a representation that matches the geometry better or incorporates conserved symmetries of the system will lead to a greatly simplified description, reveal the essential physics more clearly and make numerical simulations efficient. Thus, we will decompose the quantum field in the position representation $|\mathbf{x}\rangle$

$$
\hat{a}_{\mathbf{x}}=\sum_{i_{1}, j_{1}, k_{1}}\left\langle\mathbf{x} \mid i_{1}, j_{1}, k_{1}\right\rangle \hat{a}_{i_{1}, j_{1}, k_{1}} \equiv \sum_{1}\langle\mathbf{x} \mid 1\rangle \hat{a}_{1}
$$

in another complete, yet unspecified basis $\left\{|1\rangle \equiv\left|i_{1}, j_{1}, k_{1}\right\rangle\right\}$, which is supposedly more suitable. Furthermore, we will also employ an implicit summation convention over the quantum labels necessary to specify a state completely. This means that summation symbols are omitted and a repeated occurrence of a dummy summation index on one side of an equation implies a summation.

In this generic basis, the dilute gas Hamiltonian of Eq. (1) reads

$$
\hat{H}=\mathcal{H}^{12} \hat{a}_{1}^{\dagger} \hat{a}_{2}+\phi^{1234} \hat{a}_{1}^{\dagger} \hat{a}_{2}^{\dagger} \hat{a}_{3} \hat{a}_{4}
$$

where we have introduced the matrix elements

$$
\mathcal{H}^{12}=\langle 1|\mathcal{H}| 2\rangle=\int d^{3} x\langle 1 \mid \mathbf{x}\rangle \mathcal{H}(\mathbf{x})\langle\mathbf{x} \mid 2\rangle,
$$

of the single particle Hamiltonian Eq. (3) and the two-particle matrix elements

$$
\begin{aligned}
\phi^{1234} & =\frac{1}{2}(\mathcal{S})\left\langle 1\left|\otimes\left\langle 2\left|V_{\mathrm{bin}}\left(\mathbf{x}_{1}-\mathbf{x}_{2}\right)\right| 3\right\rangle \otimes\right| 4\right\rangle \\
& =\phi^{1243}=\phi^{2134}=\phi^{2143} \\
& =\frac{1}{!}_{4}^{3}
\end{aligned}
$$

from the binary interaction potential. Due to the bosonic nature of the particles, only the symmetric part $(\mathcal{S})$ of the instantaneous coupling vertex $\phi^{1234}$ is physically relevant and the diagrammatic representation also carries this property.

In the low kinetic energy range that we are interested in, repulsive s-wave scattering is the dominant two-particle scattering event $[44,45]$. Provided that a proper T-matrix scattering calculation has been performed $[39,46]$ or one has obtained the experimental scattering data, one can encode this information efficiently via a pseudo potential method [47, 48]. In the most elementary invocation of the method, one uses a fictitious contact potential $V_{\text {bin }}\left(\mathbf{x}_{1}, \mathbf{x}_{2}\right)=V_{0} \delta\left(\mathbf{x}_{1}-\mathbf{x}_{2}\right)$ with a single parameter $V_{0}$. This parameter is directly related to the scattering length $a_{\mathrm{s}}$ of two particles in vacuo by $V_{0}=4 \pi \hbar^{2} a_{\mathrm{s}} / m$, provided we would limit all physical approximations to a first order contribution in $V_{0}$. Otherwise again an infinite order resummation takes place and will lead to a renormalization of the effective coupling constant $V_{0}$. We will demonstrate this renormalization of the effective coupling constant takes place in a self-consistent calculation and also leads to a natural momentum cut-off. In the case of such a contact potential, one finds for the two-body matrix elements:

$$
\phi^{1234}=\frac{V_{0}}{2} \int d^{3} x\langle 1 \mid \mathbf{x}\rangle\langle 2 \mid \mathbf{x}\rangle\langle\mathbf{x} \mid 3\rangle\langle\mathbf{x} \mid 4\rangle,
$$


which need not be symmetrized, as it is symmetric already.

In general, no exact solutions of the field equation Eq. (3) or eigen-states of the Hamiltonian Eq. $(1,6)$ are known and the few celebrated exceptions [49] such as the Tonks-Girardeau gas [18, 50], the Lieb-Lininger solution [37], the Richardson pairing model [51, 52] and fermionic Luttinger liquids [53] serve as testing grounds to prove the approximation schemes. Fortunately however, most physical phenomena are of universal character. Thus the system under investigation does not have to follow precisely a particular model to show a certain response and various approximations are admissible as long as the main universal aspects of the problem are incorporated in the specific model hypothesis.

\section{B. Reduced state description with master variables}

The method of quasi-averages and self-consistent field equations has a long standing tradition in the description of classical gases and fluids [54], in plasma physics, nuclear matter physics [55] and condensed matter physics [56-59]. It has been applied successfully to classical particles and degenerate superfluid bosonic as well as fermionic systems.

The basic premises for a reduced state description of a weakly correlated many-body problem relies on the existence of a well separated hierarchy of time, energy and length scales. If this is the case, one can assume that the information required to describe an ensemble effectively can be parameterized with a set of a few relevant variables [56]. However the price that has to be payed for reducing the "astronomical" dimension of the linear many-body Schrödinger equation is giving up the superposition principle and embarking on nonlinear mathematics.

In the mean-field approximation, we want to assume that the ensemble of relevant quantum states establishes a well defined mean value for the field operator and that quantum fluctuations only cause small deviations around it

$$
\hat{a}_{1}=\left\langle\hat{a}_{1}\right\rangle+\delta \hat{a}_{1}
$$

such that $\left\langle\delta \hat{a}_{1}\right\rangle=0$. As mentioned before in Eq. (5), we use in here the shorthand notation for any complete set quantum of labels e.g., $1 \equiv \mathrm{x}_{1}$ in position space or $1 \equiv k_{1}$ in momentum space, respectively. This number symmetry breaking approximation is tremendously useful and can be envisaged also as the semi-classical limit of coherent many-particle quantum physics in analogy to the description of the optical laser [60]. However, one must be aware of the implied consequences that have been discussed in the literature $[2,23,26,61]$ and number conserving approximations [62-64] have their merits, but shortcomings as well.

Based on these assumptions, we want to sum the c-number field amplitudes $\alpha_{1}=\left\langle\hat{a}_{1}\right\rangle$ that were introduced in Eq. (10) over this complete set of states and form a basis-independent element of a Hilbert space as

$$
|\alpha\rangle=\alpha_{1}|1\rangle \equiv\langle\hat{a}\rangle=\sim
$$

In here, we have used the Dirac notation with the implied standard scalar product $\langle\alpha \mid \beta\rangle=\int d^{3} x \alpha^{*}(\mathbf{x}) \beta(\mathbf{x})$. Due to the non-linear nature of the ensuing mean field equations, we can no longer rely on the dynamical superposition principle available in linear quantum mechanics. However, the advantage of the notation arises from preserving and emphasizing the geometrical transformations properties of all correlations functions under a change of basis or, most generally, frame of reference. The wiggly line has been introduced to represents the mean-field amplitude graphically. This symbol literally denotes the state at time $t$ and not the time-ordered propagator that evolves it.

In an analogous fashion, we can separate the single particle density operator of the atomic gas $f=\left\langle\hat{a}^{\dagger} \hat{a}\right\rangle=f^{(c)}+\tilde{f}$ into a mean-field contribution $f^{(c)}$ and a fluctuation $\tilde{f}$ around it. This contribution $\tilde{f}$ is also known as the normal quantum depletion of the atomic cloud. Both quantities are hermitian tensor operators of rank $(1,1)$ and defined as

$$
f^{(c)}=|\alpha\rangle\left\langle\alpha\left|, \quad \tilde{f}=\tilde{f}_{14}\right| 1\right\rangle\langle 4|=\longleftarrow .
$$

For example, in a position representation, the single particle density matrix reads as $\left\langle\mathbf{x}_{1}|f| \mathbf{x}_{2}\right\rangle=f^{(c)}\left(\mathbf{x}_{1}, \mathbf{x}_{2}\right)+$ $\tilde{f}\left(\mathbf{x}_{1}, \mathbf{x}_{2}\right)=\alpha\left(\mathbf{x}_{1}\right) \alpha^{*}\left(\mathbf{x}_{2}\right)+\left\langle\left(\hat{a}_{\mathbf{x}_{2}}^{\dagger}-\alpha^{*}\left(\mathbf{x}_{2}\right)\right)\left(\hat{a}_{\mathbf{x}_{1}}-\alpha\left(\mathbf{x}_{1}\right)\right)\right\rangle$

Similarly, we define anomalous averages or pairing fields $m=\langle\hat{a} \hat{a}\rangle=m^{(c)}+\widetilde{m}$, as symmetric tensors of rank $(2,0)$, i.e.,

$$
m^{(c)}=|\alpha\rangle|\alpha\rangle, \quad \widetilde{m}=\widetilde{m}_{12}|1\rangle|2\rangle=\hookrightarrow,
$$

as well as their symmetric conjugates as $\widetilde{m}^{*}=\longrightarrow \leftarrow$. These anomalous averages are also associated with the quantum depletion of the degenerate gas. In contrast to the single particle nature of normal density matrix of Eq. (12), it can be seen immediately from the structure of Eq. (13) that the pairing field is a two-particle state. It will be shown in Sec. II $\mathbf{F}$ that the pairing field $\widetilde{m}\left(\mathbf{x}_{1}, \mathbf{x}_{2}\right)$ evolves basically like a generalization the bare two-particle Schrödinger state. Thus, it carries all the important physics of binary scattering. In a position representation, the pairing field is given by $\left\langle\mathbf{x}_{1}\right|\left\langle\mathbf{x}_{2}\right| m=m^{(c)}\left(\mathbf{x}_{1}, \mathbf{x}_{2}\right)+\widetilde{m}\left(\mathbf{x}_{1}, \mathbf{x}_{2}\right)=\alpha\left(\mathbf{x}_{1}\right) \alpha\left(\mathbf{x}_{2}\right)+$ $\left\langle\left(\hat{a}_{\mathbf{x}_{2}}-\alpha\left(\mathbf{x}_{2}\right)\right)\left(\hat{a}_{\mathbf{x}_{1}}-\alpha\left(\mathbf{x}_{1}\right)\right)\right\rangle$.

\section{Structure of the generalized density matrix}

From the transformation properties of the matrices under coordinate change, one finds that the normal density matrix $\tilde{f}(t)$ and the pairing fields $\widetilde{m}(t)$ are not independent but actually the components of a generalized density matrix $G$ $[55,58,65]$. If we represent the system in a state space of dimension $n$, then it is convenient to arrange the mean field in a $2 \mathrm{n}$-dimensional row vector $\chi$ and the fluctuations as a positive semi-definite matrix $\operatorname{dim}[G]=2 n \times 2 n$,

$$
\chi=\left(\begin{array}{c}
\alpha \\
\alpha^{*}
\end{array}\right), \quad G=\left(\begin{array}{cc}
\tilde{f} & \tilde{m} \\
\tilde{m}^{*} & (\mathbb{1}+\tilde{f})^{*}
\end{array}\right) \geq 0 .
$$


The non-negativity of this co-variance matrix implies that the magnitude of the anomalous fluctuations is limited by the normal depletion through a Cauchy-Schwartz inequality (see Appendix A). In the general context of Green function's, this single-time density operator $G(t)$ can also be viewed as a particular limit of a time-ordered $(\mathcal{T})$, two-time Green function $G(\tau, t)$, i. e., $G(t) \equiv G^{>}(t)=\lim _{\tau \rightarrow t_{+}} \mathcal{T} G(\tau, t)$. Consequently, it is also necessary to consider the opposite limit and to define a time-reversed, single-time density operator through $G^{<}(t)=\lim _{\tau \rightarrow t_{-}} \mathcal{T} G(\tau, t)$. Explicitly, this operator is given by

$$
G^{<}=\sigma_{1} G^{>*} \sigma_{1}=G^{>}+\sigma_{3}=\left(\begin{array}{cc}
\mathbb{1}+\tilde{f} & \tilde{m} \\
\widetilde{m}^{*} & \tilde{f}^{*}
\end{array}\right),
$$

where standard Pauli spin matrices have been introduced and are defined in Appendix B.

The specific structure of the generalized density matrix implies various important physical properties. First of all, we have to assume that there is a basis that diagonalizes this fluctuation matrix. Exactly $n$ of its $2 n$ eigen-values correspond to the positive occupation numbers of finding a particle or, more generally, a quasi-particle in a certain mode. For a given, but otherwise arbitrary, $G$ matrix, one can construct this basis by studying the transformation law of the density matrix under a canonical transformation $T$ (see Appendix C),

$$
G^{\prime}=T G T^{\dagger}
$$

It is important to note that this is not the transformation law of a general matrix under coordinate change. This would require that $T^{\dagger}=T^{-1}$. However, by only using the properties of the symplectic transformations, one can show that a canonical eigen-value problem is defined by

$$
\left(\sigma_{3} G\right) T^{\dagger}=T^{\dagger}\left(\sigma_{3} G^{\prime}\right)
$$

The solution of this eigen-value problem yields the eigenvector matrix $T^{\dagger}$ and the corresponding diagonal eigen-value matrix $\sigma_{3} G^{\prime}$. All normalizable states can be rescaled such that $T \sigma_{3} T^{\dagger}=\sigma_{3}$. Now, we are able to reconstruct the positive $G$ matrix

$$
G=V P V^{\dagger}
$$

from its eigen-vectors $V=\sigma_{3} T^{\dagger}$ and the diagonal, positive occupation number matrix $P=\sigma_{3} G^{\prime} \sigma_{3}$.

Second, an important feature of an admissible fluctuation matrix is its consistency with the commutation relation, i.e. , $\left\langle\hat{a}_{1} \hat{a}_{2}^{\dagger}\right\rangle=\left\langle\hat{a}_{2}^{\dagger} \hat{a}_{1}\right\rangle+\delta_{12}$ and $\left\langle\hat{a}_{1} \hat{a}_{2}\right\rangle=\left\langle\hat{a}_{2} \hat{a}_{1}\right\rangle$. This was already expressed in Eq. (15) as

$$
\sigma_{1} G^{*} \sigma_{1}-G=\sigma_{3}
$$

By invoking the properties of a unitary symplectic transformation, one can show that the elements of the diagonal occupation number matrix $P$ are not $2 n$ independent variables. Actually half of them are determined by the other half, $P_{(n+1, \ldots, 2 n)}=1+P_{(1, \ldots, n)}$, or

$$
\sigma_{1} P \sigma_{1}-P=\sigma_{3} .
$$

In other words, by separating the occupation numbers $P$ and the eigen-vector matrix $V$ into a first and second half, i.e., $P=\left(P_{+}, \mathbb{1}+P_{+}\right)$and $V=\left(V_{+}, V_{-}\right)$, one can then decompose a general fluctuation matrix as

$$
G=V_{+} P_{+} V_{+}^{\dagger}+V_{-}\left(\mathbb{1}+P_{+}\right) V_{-}^{\dagger} .
$$

\section{Dynamic equations of motion}

It is now straight forward to derive equations of motion for these averages directly from the Heisenberg equation Eq. (3). However, due to the nonlinear character of the operator equation, one finds always a coupling of correlation functions involving $n$ fields to a correlation function of $n+2$ fields. If we truncate this infinite hierarchy of correlation functions (BBGKY) at the level of one and two-operator fields $\{\alpha, \tilde{f}, \widetilde{m}\}$ and approximate higher correlation functions with the help of Wick's theorem [27, 28, 56], one obtains the following equations of motion, also known as time-dependent Hartree-Fock Bogoliubov (THFB) equations (THFB)

$$
\begin{aligned}
i \hbar \frac{d}{d t} \chi & =\Pi \chi, \\
i \hbar \frac{d}{d t} G & =\Sigma G-G \Sigma^{\dagger} .
\end{aligned}
$$

For the evolution of the mean-field $\chi$, one finds a generalized Gross-Pitaevskii propagator that is defined as

$$
\Pi=\left(\begin{array}{cc}
\Pi_{\mathcal{N}} & \Pi_{\mathcal{A}} \\
-\Pi_{\mathcal{A}}^{*} & -\Pi_{\mathcal{N}}^{*}
\end{array}\right)
$$

The two contributions that define this symplectic propagator are a normal hermitian Hamiltonian operator and a symmetric anomalous coupling potential

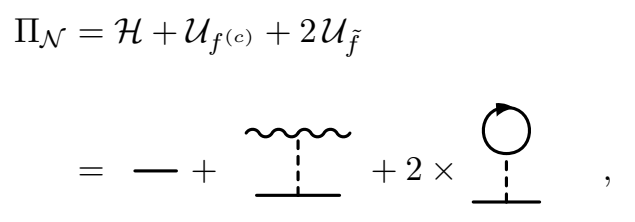

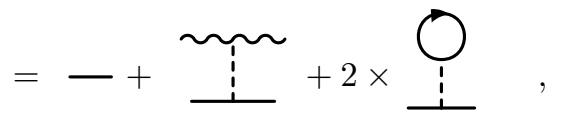

$$
\Pi_{\mathcal{A}}=\mathcal{V}_{\tilde{m}}=
$$

It is easy to identify $\Pi_{\mathcal{N}}$ with the well known hermitian GPpropagator that accounts for the free evolution of the meanfield $\mathcal{H}$, its self-interaction $\mathcal{U}_{f(c)}$, as well as the energy shift $\mathcal{U}_{\tilde{f}}$, which caused by the presence of the non-condensate cloud. However, due to the existence of the anomalous fluctuations there is also a coupling through $\Pi_{\mathcal{A}}$ to the time-reversed field. For convenience, we have introduced two auxiliary potentials $\mathcal{U}_{f}$ and $\mathcal{V}_{m}$. Explicitly, they are defined in terms of the two-body matrix elements as

$$
\begin{aligned}
& \mathcal{U}_{f}^{14}=2 \phi^{1234} f_{32}, \\
& \mathcal{V}_{m}^{12}=2 \phi^{1234} m_{34} .
\end{aligned}
$$


In a position representation, this reduces to the familiar nonlocal Hartree-Fock potentials

$$
\begin{aligned}
\mathcal{U}_{f}(\mathbf{x}, \mathbf{y}) & =\frac{1}{2}\left[V_{\text {bin }}(\mathbf{x}-\mathbf{y}) f(\mathbf{x}, \mathbf{y})\right. \\
& \left.+\delta(\mathbf{x}-\mathbf{y}) \int d^{3} z V_{\text {bin }}(\mathbf{z}-\mathbf{y}) f(\mathbf{z}, \mathbf{z})\right], \\
\mathcal{V}_{m}(\mathbf{x}, \mathbf{y}) & =V_{\text {bin }}(\mathbf{x}-\mathbf{y}) m(\mathbf{x}, \mathbf{y}) .
\end{aligned}
$$

Similarly, one finds that the evolution of the density operator $G$ is ruled by a HFB self-energy $\Sigma$ that can be obtained also by variational methods [58]. In detail, this symplectic self-energy is given by

$$
\Sigma=\left(\begin{array}{cc}
\Sigma_{\mathcal{N}} & \Sigma_{\mathcal{A}} \\
-\Sigma_{\mathcal{A}}^{*} & -\Sigma_{\mathcal{N}}^{*}
\end{array}\right)
$$

where we have introduced hermitian Hamiltonian operators and symmetric anomalous coupling potentials as

$$
\begin{aligned}
& \Sigma_{\mathcal{N}}=\mathcal{H}+2 \mathcal{U}_{f^{(c)}}+2 \mathcal{U}_{\tilde{f}}
\end{aligned}
$$

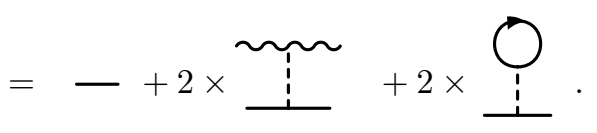

$$
\begin{aligned}
& \Sigma_{\mathcal{A}}=\mathcal{V}_{m^{(c)}}+\mathcal{V}_{\tilde{m}}=\overbrace{}^{n}+
\end{aligned}
$$

It is important to note the different weighing factors of the mean-field potential in Eqs. (25) and (32) and the fact that the potentials are local in time.

\section{E. Structure of the Hartree-Fock-Bogoliubov self energy}

\section{Normal quasi-particle modes}

Symplectic self-energy operators arises not only naturally in kinetic theories $[28,66]$ or variational calculations, but in many other contexts involving stability analysis. In the case of bosonic fields, the self-energy operator is of the generic form:

$$
\Sigma=\left(\begin{array}{cc}
\Sigma_{\mathcal{N}} & \Sigma_{\mathcal{A}} \\
-\Sigma_{\mathcal{A}}^{*} & -\Sigma_{\mathcal{N}}^{*}
\end{array}\right)
$$

In here, $\Sigma_{\mathcal{N}}$ stands for a hermitian operator $\Sigma_{\mathcal{N}}=\Sigma_{\mathcal{N}}^{\dagger}$ and $\Sigma_{\mathcal{A}}$ denotes an anomalous coupling term that has to be symmetric $\Sigma_{\mathcal{A}}=\Sigma_{\mathcal{A}}^{\top}$. The relative size of the operators $\Sigma_{\mathcal{N}}$ and $\Sigma_{\mathcal{A}}$ determines the character of the energy spectrum. It can either be real valued with pairs of positive and negative eigen-energies, or one finds a doubly degenerate zero eigenvalue, if the energy difference between the smallest positive and highest negative vanishes (gap-less spectrum). In the general case, there is a mixed spectrum consisting of pairs of real sign-reversed as well as pairs of complex conjugated eigenvalues. The eigen-vectors $W$ are normalizable with respect to the indefinite norm $\|W\|^{2}=W^{\dagger} \sigma_{3} W$, except for those that belong to zero or complex eigen-values. It is important to note that this energy basis $W$ is in general distinct from the instantaneous basis $V$ that diagonalizes the fluctuation matrix $G$ in Eq. (18). They do coincide only in equilibrium. The mathematical properties of the eigen-states $W$ can be derived easily from the intrinsic symmetries of the HFB self-energy operator:

$$
\begin{aligned}
\Sigma & =-\sigma_{1} \Sigma^{*} \sigma_{1}, \\
\Sigma^{\dagger} & =\sigma_{3} \Sigma \sigma_{3} .
\end{aligned}
$$

Thus, if $W$ is a solution of the right eigen-value problem with energy $E$,

$$
\Sigma W=W E,
$$

it follows directly from Eq. (35) that $\bar{W}=\sigma_{1} W^{*}$, is also a right eigen-vector but corresponds to the eigen-value $\bar{E}=$ $-E^{*}$. Starting from the second symmetry in Eq. (36) and the right eigen-value problem of Eq. (37), it is easy to construct the left eigen-vectors $\widetilde{W}=W^{\dagger} \sigma_{3}$ that correspond to the eigen-values $\tilde{E}=E^{*}$ :

$$
\widetilde{W} \Sigma=E^{*} \widetilde{W} .
$$

Finally, from a combination of the results for the right and left eigen-vectors, it follows that the eigen-vectors are orthogonal with respect to the metric $\sigma_{3}$ :

$$
0=\left(E^{*}-E^{\prime}\right) W_{E}^{\dagger} \sigma_{3} W_{E^{\prime}},
$$

if $E^{*} \neq E^{\prime}$. On the other hand, this relation implies also that eigen-vectors that belong to complex eigen-values must have zero norm.

\section{Defective sub-spaces}

The situation of a doubly degenerate zero energy eigenvalue $E=0$ needs special attention. One can view this case as a limit when two non-degenerate states approach each other. However, as the energy gap decreases, the two eigen-states become more and more collinear. Thus, in the limit of a vanishing energy separation, the dimension of the spanned vector space collapses from 2 to 1 and $\Sigma$ becomes defective [58, 67]. In the present context, a gap-less linear response matrix occurs from a perturbation analysis of the simple Gross-Pitaevskii equation and describes the collective excitation of the system. The emerging zero energy state is called Goldstone mode and can be interpreted physically as an attempt to restore the broken number symmetry. On the other hand, we find for the THFB Eqs. $(22,23)$ that the self-energy matrix $\Sigma$ has a gap and its eigen-states form a complete non-defective basis. It is important to distinguish these states from the collective excitations of the total system, whose excitation spectrum is again gap-less $[29,68]$.

The general situation can be described by separating the two ground state modes $\left(W_{E_{0}}, W_{-E_{0}}\right)$ from the remaining 
states $W^{\prime}$ such that $\operatorname{dim}\left[W^{\prime}\right]=2 n \times 2(n-1)$ and the diagonal eigenvalue matrix has a $\operatorname{dim}\left[E^{\prime}\right]=2(n-1) \times 2(n-1)$. By introducing two quadrature modes $P Q$ with $\operatorname{dim}[P Q]=2 n \times 2$ via

$$
\begin{aligned}
\Sigma W^{\prime} & =W^{\prime} E^{\prime}, \\
\Sigma P Q & =P Q i \hbar\left(\begin{array}{cc}
0 & -M^{-1} \\
M \omega_{0}^{2} & 0
\end{array}\right),
\end{aligned}
$$

one can span a two dimensional vector space which is orthogonal to the higher modes $W^{\prime}$ and does not collapse $[69,70]$. This is mathematically achieved by the construction of a "best" basis in the context a singular value decomposition [67] and introduces two singular values, i. e., an inertial mass parameter $M$ and a gap energy $E_{0}=\hbar \omega_{0}$ of the number and phase quadratures. The quadrature states satisfy the following orthogonality

$$
P Q^{\dagger} \sigma_{3} W^{\prime}=0, \quad P Q^{\dagger} \sigma_{3} P Q=\hbar \sigma_{2}^{(1)},
$$

and symmetry relations $P Q=-\sigma_{1} P Q^{*}$. From a dimensional consideration, it is obvious that one also has to use lower dimensional Pauli matrices, which act in the appropriate subspaces such that $\operatorname{dim}\left[\sigma_{k=1,2,3}^{(l)}\right]=2 l \times 2 l$. All states together form again a complete basis such that

$$
W^{\prime} \sigma_{3}^{(n-1)} W^{\prime \dagger}+P Q \frac{\sigma_{2}^{(1)}}{\hbar} P Q^{\dagger}=\sigma_{3}
$$

With these states, we can then obtain the following spectral decomposition of the self-energy

$$
\begin{aligned}
& \Sigma \sigma_{3}=W^{\prime}\left(E^{\prime} \sigma_{3}^{(n-1)}\right) W^{\prime \dagger}+P Q\left(\begin{array}{cc}
M^{-1} & 0 \\
0 & M \omega_{0}^{2}
\end{array}\right) P Q^{\dagger} \\
& =W^{\prime}\left(E^{\prime} \sigma_{3}^{(n-1)}\right) W^{\prime \dagger}+\frac{1}{M} P \cdot P^{\dagger}+M \omega_{0}^{2} Q \cdot Q^{\dagger} .
\end{aligned}
$$

The physical meaning of the quadrature states can be understood most clearly when mapping them again back onto the quantum field

$$
\left(\begin{array}{c}
\hat{P} \\
\hat{Q}
\end{array}\right)=P Q^{\dagger} \sigma_{3}\left(\begin{array}{c}
\hat{a} \\
\hat{a}^{\dagger}
\end{array}\right),\left(\begin{array}{c}
\hat{b} \\
\hat{b}^{\dagger}
\end{array}\right)=W^{\prime \dagger} \sigma_{3}\left(\begin{array}{c}
\hat{a} \\
\hat{a}^{\dagger}
\end{array}\right) .
$$

Now, these field quadratures $\hat{Q}, \hat{P}$ satisfy a conventional Heisenberg-Weyl algebra and define in depend, as well as orthogonal bosonic quasi-particles $\hat{b}_{i}$ according to

$$
[\hat{Q}, \hat{P}]=i \hbar, \quad\left[\hat{b}_{i}, \hat{b}_{j}^{\dagger}\right]=\delta_{i j}
$$

\section{F. Upgrading off-diagonal potentials to many-body T-matrices}

While one can understand the physical structure of the self-energy best in the general form of the kinetic equations Eq. (23), one can appreciate other aspects much better by considering the equations for the components individually.

$$
\begin{gathered}
i \hbar \frac{d}{d t} \tilde{f}=\Sigma_{\mathcal{N}} \tilde{f}-\tilde{f} \Sigma_{\mathcal{N}}+\Sigma_{\mathcal{A}} \widetilde{m}^{*}-\widetilde{m} \Sigma_{\mathcal{A}}^{*}, \\
i \hbar \frac{d}{d t} \widetilde{m}=\Sigma_{\mathcal{N}} \tilde{m}+\widetilde{m} \Sigma_{\mathcal{N}}^{*}+\Sigma_{\mathcal{A}}(\mathbb{1}+\tilde{f})^{*}+\tilde{f} \Sigma_{\mathcal{A}},
\end{gathered}
$$

In stationarity and by assuming a real valued self-energy, one can solve for $\widetilde{m}$ in an HF eigen basis

$$
\Sigma_{\mathcal{N}}\left|\epsilon_{i}\right\rangle=\left(\epsilon_{i}-\mu\right)\left|\epsilon_{i}\right\rangle
$$

and finds

$$
\widetilde{m}_{12}=\frac{\Sigma_{\mathcal{A}}^{13}(\mathbb{1}+\tilde{f})_{32}^{*}+\tilde{f}_{13} \Sigma_{\mathcal{A}}^{32}}{2 \mu-\left(\epsilon_{1}+\epsilon_{2}\right)}
$$

No rules for circumventing poles in the above energy denominator need to be specified as all energies satisfy $\epsilon_{i}>\mu$.

In the present article, we have only considered first order processes and found the one-loop contribution to the selfenergy as

$$
\Sigma_{\mathcal{A}}=\mathcal{V}_{m^{(c)}}+\mathcal{V}_{\widetilde{m}}=\overbrace{1}+
$$

Now, if we substitute the stationary solution of Eq. (50) into this equation, one obtains an inhomogeneous linear equation for the self-energy

$$
\Sigma_{\mathcal{A}}^{12}=2 \phi^{1234} m_{34}^{(c)}+2 \phi^{1234} \frac{(\mathbb{1}+2 \tilde{f})_{33^{\prime}}}{2 \mu-\left(\epsilon_{3}+\epsilon_{4}\right)} \Sigma_{\mathcal{A}}^{3^{\prime} 4} .
$$

It is now clear that the value of $\Sigma_{\mathcal{A}}$ is only determined by $m^{(c)}$ in the absence of a homogeneous contribution. Thus, there must be a T-matrix that maps mean-field to the off-diagonal self-energy

$$
\Sigma_{\mathcal{A}}^{12}=T_{\mathcal{A}}^{1234}(2 \mu) m_{34}^{(c)}=\overbrace{1}^{2}
$$

This leads to the following general definition for an off-the energy shell T-matrix

$$
T_{\mathcal{A}}^{1234}(\epsilon)=2 \phi^{1234}+2 \phi^{123^{\prime} 4^{\prime}} \frac{(\mathbb{1}+2 \tilde{f})_{3^{\prime} 3^{\prime \prime}}}{\epsilon-\left(\epsilon_{3^{\prime}}+\epsilon_{4^{\prime}}\right)} T_{\mathcal{A}}^{3^{\prime \prime} 4^{\prime} 34}
$$

In operator notation this is equivalent to

$$
T_{\mathcal{A}}(\epsilon)=V+V G_{\mathcal{A}}(\epsilon)[(\mathbb{1}+2 \tilde{f}) \otimes \mathbb{1}] T_{\mathcal{A}}(\epsilon)
$$

where $G_{\mathcal{A}}(\epsilon)=\left(\epsilon-\Sigma_{\mathcal{N}} \otimes \mathbb{1}-\mathbb{1} \otimes \Sigma_{\mathcal{N}}\right)^{-1}$ is a Green's function, which describes the propagation of two independent HF particles according to Eq. (49). The diagrammatic representation of this $T_{\mathcal{A}}$-matrix relation is

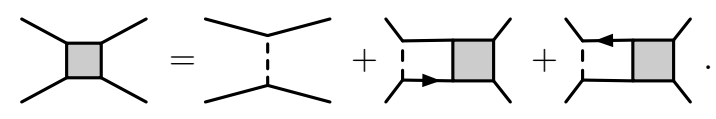




\section{G. Invariants and Conservation laws}

\section{Number}

The total particle number $\hat{N}=\int d^{3} x \hat{a}_{\mathbf{x}}^{\dagger} \hat{a}_{\mathbf{x}}$ is a conserved quantity if the atoms evolve under the generic two-particle Hamiltonian operator $\hat{H}$ given by Eq. (1), i. e., $[\hat{H}, \hat{N}]=0$. This conservation law implies that the system is invariant under a global phase change $\hat{a} \rightarrow \hat{a} \exp (-i \Phi)$. By using this continuous symmetry, i. e., $\alpha \rightarrow \alpha \exp (-i \Phi), \tilde{f} \rightarrow \tilde{f}$, and $\widetilde{m} \rightarrow \widetilde{m} \exp (-2 i \Phi)$, it is easy to see that the kinetic equations Eqs. $(22,23)$ are also explicitly number conserving at all times:

$$
N(t)=\langle\hat{N}\rangle=\operatorname{Tr}\left\{f^{(c)}(t)+\tilde{f}(t)\right\}=\text { const. }
$$

Nevertheless, it is important to note that out-of equilibrium there can be a continuous particle exchange between the condensate and the non-condensate clouds.

\section{Energy}

In the absence of any time-dependent external driving fields, such as optical lasers or magnetic rf-fields, the overall energy $\hat{H}$ must be conserved as well. To find the expectation value of the total system energy $E=\langle\hat{H}\rangle$, we use again Wick's theorem (see Appendix E) systematically. Explicitly, this energy functional is given as

$$
\begin{aligned}
& E(t)=\operatorname{Tr}\left\{\left[\mathcal{H}+\frac{1}{2} \mathcal{U}_{f(c)}+\mathcal{U}_{\tilde{f}}\right] f^{(c)}+\left[\mathcal{H}+\mathcal{U}_{f(c)}+\mathcal{U}_{\tilde{f}}\right] \tilde{f}\right. \\
& \left.+\frac{1}{2} \mathcal{V}_{\tilde{m}} m^{(c)^{*}}+\frac{1}{2}\left[\mathcal{V}_{m^{(c)}}+\mathcal{V}_{\tilde{m}}\right] \widetilde{m}^{*}\right\}=\text { const. }
\end{aligned}
$$

That this is also a constant of motion follows straight from Eqs. $(22,23)$ and make this a "derivable theory" according to Ref. [23]. For example, the same first order results can be found in Ref. [58], derived by a variational procedure.

\section{Entropy and individual occupation probabilities}

In a previous section Sec. II C, we have demonstrated with Eq. (18) that any admissible covariance matrix is necessarily of the following form

$$
\begin{aligned}
G(t) & =V(t) P(t) V(t)^{\dagger} \\
\sigma_{3} & =V(t)^{\dagger} \sigma_{3} V(t) .
\end{aligned}
$$

Furthermore, we have derived a non-linear equation of motion (23) for this entity in Sec. IID. It is not self-evident that the aforementioned constraints on the structure of the density matrix are automatically preserved during the time evolution. Fortunately however, this is the case. This can be verified easily by inserting Eq. (59) into Eq. (23). From this, one finds that all instantaneous eigenvalues of the density matrix are constants of motion

$$
P(t)=\text { const. }
$$

provided that the instantaneous eigen basis evolves according to

$$
i \hbar \frac{d}{d t} V(t)=\Sigma V
$$

In turn, this is compatible with Eq. (60) due to the symmetry of the self-energy in Eq. (36).

\section{RESULTS}

\section{A. Rescaling the kinetic equations to a prolate, quasi one-dimensional configuration}

General discussions on the properties of many-body physics are usually plagued by a very abstract language. In order to gain further insight into the complex non-linear physics, it is mostly necessary to examine spatially homogeneous systems and assume stationary in the time-domain. This makes it possible to continue with analytical work and to capture the essential bulk physics of macroscopic systems. However, trapped quantum gases are different in many respects as the system size is of the same order as the coherence length, thus boundary effects are of equal importance. Moreover, it is difficult to discuss succinctly the meaning of the thermodynamic limit in a quantum system with a mesoscopic particle number and a discrete energy spectrum.

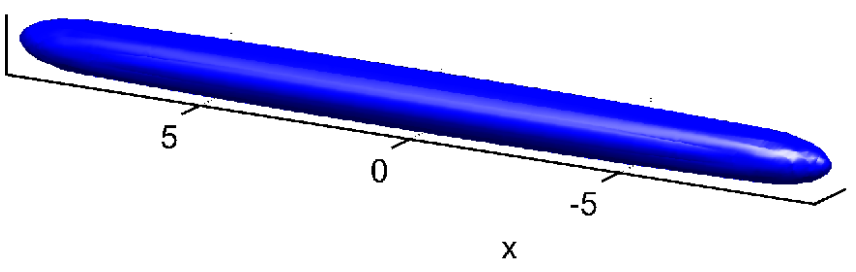

Figure 1: Schematic representation of the density distribution in a typical "cigar-shaped" trapped gas. The kinematic motion in the orthogonal directions $y, x$ is frozen out and presumably all the dynamics occurs along the weakly trapped $x$-axis.

In the following sections, we will therefore examine numerically the properties of a quasi-one dimensional prolate system as shown in Fig. 1. While we have studied already a three-dimensional isotropic configuration [71], prolate "cigar-shaped" traps are currently at the focus of attention $[40,43,72,73]$. Due to the reduction of the available phasespace volume, the role of quantum fluctuations becomes more pronounced in low dimensional systems.

To be specific, we want to assume that the single particle 
trap Hamiltonian of Eq. (4) is of the form

$$
\begin{gathered}
\mathcal{H}(\mathbf{x})=-\frac{\hbar^{2}}{2 m} \Delta+V_{\text {ho }}(\mathbf{x})-\mu, \\
V_{\text {ho }}(\mathbf{x})=\frac{1}{2} m \omega^{2} x^{2}+\frac{1}{2} m \omega_{\perp}^{2}\left(y^{2}+z^{2}\right) .
\end{gathered}
$$

A measure of the anisotropy of this trap geometry is the aspect ratio $\beta=\omega_{\perp} / \omega$. In a very elongated, prolate configuration the perpendicular oscillation frequency $\omega_{\perp}$ is much larger than the longitudinal circular frequency $\omega$, thus $\beta \gg 1$. In order to reduce the kinetic equations also to a dimensionless form, we choose the ground state extension of the longitudinal harmonic oscillator $a_{0}=\sqrt{\hbar / m \omega}$ as the basic length scale, $t_{0}=2 \pi / \omega$ as the basic time-scale and $E_{0}=\hbar \omega$ as the natural energy unit.

The present formulation of the theory in terms of a generic set of basis states $\{|1\rangle\}$ is able to handle the dimensional reduction from the general three-dimensional kinetic theory to the quasi one-dimensional situation quite easily, if we use an adapted trap basis in terms of longitudinal and perpendicular harmonic oscillator states $|1\rangle=\left|i_{1}\right\rangle \otimes\left|j_{1}, k_{1}\right\rangle_{\perp}$. By assuming that only the ground state components of the fields in the transverse directions are occupied, one can limit the evolution to one dimension effectively

$$
\begin{gathered}
|\alpha\rangle=\alpha_{1}|1\rangle \otimes|0,0\rangle_{\perp} \\
\tilde{f}=\tilde{f}_{1,2}|1\rangle\langle 2|\otimes| 0,0\rangle_{\perp}\left\langle 0,\left.0\right|_{\perp},\right. \\
\tilde{m}=\tilde{m}_{1,2}|1\rangle|2\rangle \otimes|0,0\rangle_{\perp}|0,0\rangle_{\perp} .
\end{gathered}
$$

In order to evaluate the matrix elements of the binary interaction potential further, we need the position representation of the normalized two-dimensional harmonic oscillator groundstate

$$
\begin{gathered}
\langle y, z \mid 0,0\rangle=\varphi_{0}(y, z ; t)=e^{-i \beta \omega t} \sqrt{\frac{\beta}{\pi a_{0}^{2}}} e^{-\beta \frac{y^{2}+z^{2}}{2 a_{0}^{2}}}, \\
\int d y d z\left|\varphi_{0}\right|^{2}=1, \quad \int d y d z\left|\varphi_{0}\right|^{4}=\frac{\beta}{2 \pi a_{0}^{2}} .
\end{gathered}
$$

To compensate for the ground state energy of the twodimensional harmonic oscillator, we are working here with an explicitly time-dependent basis that removes that energy shift. We can now partially evaluate the matrix elements of the two-body interaction in the contact potential approximation of Eq. (9). By assuming only ground states in the transverse direction, one obtains

$$
\phi_{0}^{1234}=\hbar \omega a_{0} \frac{g}{2} \int d x\langle 1 \mid x\rangle\langle 2 \mid x\rangle\langle x \mid 3\rangle\langle x \mid 4\rangle,
$$

where we have introduced a dimensionless coupling constant $g=2 \beta a_{s} / a_{0}$. This corresponds to an effective onedimensional interaction potential $V_{\text {bin }}^{(1)}(x)=\hbar \omega a_{0} g \delta(x)$, which would produce the same matrix elements. In this limit of a very localized, point-like binary interaction, one finds further that the self-energy operator of Eqs. (24) and (31) become local operators in space and time, i.e., $\Pi(x, y, t)=$ $\delta(x-y) \Pi(x, t)$ and $\Sigma(x, y, t)=\delta(x-y) \Sigma(x, t) . \quad$ Finally, this leads to the quasi one-dimensional self-consistent Hartree-Fock-Bogoliubov (SCHFB) equations in the contact potential approximation

$$
\begin{aligned}
i \partial_{t} \chi & =\Pi(x, t) \chi(x, t) \\
i \partial_{t} G & =\Sigma\left(x_{1}, t\right) G\left(x_{1}, x_{2}, t\right)-G\left(x_{1}, x_{2}, t\right) \Sigma\left(x_{2}, t\right)^{\dagger}
\end{aligned}
$$

where local self-energies operators have been defined as follows

$$
\begin{aligned}
\Pi(x, t) & =\left(\begin{array}{cc}
\Pi_{\mathcal{N}}(x, t) & \Pi_{\mathcal{A}}(x, t) \\
-\Pi_{\mathcal{A}}^{*}(x, t) & -\Pi_{\mathcal{N}}^{*}(x, t)
\end{array}\right) \\
\Pi_{\mathcal{N}}(x, t) & =\frac{-\partial_{x}^{2}+x^{2}}{2}+g|\alpha(x, t)|^{2}+2 g \tilde{f}(x, x, t)-\mu \\
\Pi_{\mathcal{A}}(x, t) & =g \widetilde{m}(x, x, t)
\end{aligned}
$$

and

$$
\begin{aligned}
\Sigma(x, t) & =\left(\begin{array}{cc}
\Sigma_{\mathcal{N}}(x, t) & \Sigma_{\mathcal{A}}(x, t) \\
-\Sigma_{\mathcal{A}}^{*}(x, t) & -\Sigma_{\mathcal{N}}^{*}(x, t)
\end{array}\right) \\
\Sigma_{\mathcal{N}}(x, t) & =\frac{-\partial_{x}^{2}+x^{2}}{2}+2 g|\alpha(x, t)|^{2}+2 g \tilde{f}(x, x, t)-\mu \\
\Sigma_{\mathcal{A}}(x, t) & =g \alpha(x, t)^{2}+g \tilde{m}(x, x, t) .
\end{aligned}
$$

The numerical results that are presented in the following sections are based on typical ${ }^{87} \mathrm{Rb}$ condensate data, i. e., an atomic mass $m_{87}=1.443110^{-25} \mathrm{~kg}$, axial and radial trap frequencies $\left(\nu, \nu_{\perp}\right)=(3,800) \mathrm{Hz}$, which result in an aspect ratio $\beta=267$, an axial harmonic oscillator ground state size $a_{0}=6.2263 \mu \mathrm{m}$ and a $3 \mathrm{~d}$ scattering length $a_{s}=5.8209 \mathrm{~nm}$. This leads to an effective coupling constant $g=2 \beta a_{s} / a_{0}=$ 0.4986 .

\section{B. The Gross-Pitaevskii equation and the collective Bogoliubov excitations}

Before discussing the properties of the fully self-consistent equations, it is prudent to start with the most basic version of it. By disregarding $\tilde{f}$ and $\tilde{m}$ altogether, one obtains the GrossPitaevskii equation

$$
\begin{gathered}
i \partial_{t} \chi=\Pi^{\mathrm{GP}}(x, t) \chi(x, t), \\
\Pi_{\mathcal{N}}^{\mathrm{GP}}(x, t)=\frac{-\partial_{x}^{2}+x^{2}}{2}+g|\alpha(x, t)|^{2}-\mu, \Pi_{\mathcal{A}}^{\mathrm{GP}}=0 .
\end{gathered}
$$

From a stationary solution $\chi_{0}=\left(\alpha_{0}, \alpha_{0}^{*}\right)^{\top}$, which is normalized to the total particle number $N=\int d x\left|\alpha_{0}(x)\right|^{2}$, one obtains the chemical potential $\mu(N)$. In the interaction dominated mean-field regime, one may disregard the kinetic energy contribution altogether and one finds in the Thomas-Fermi approximation $\mu_{\mathrm{TF}}=(3 g N / 2)^{2 / 3} / 2$, the width of the condensate $x_{\mathrm{TF}}=\sqrt{2 \mu_{\mathrm{TF}}}$ and a healing length $\xi=1 / x_{\mathrm{TF}}$. 


\section{Linear response analysis}

From a weak perturbation around the stationary solution $\alpha(x, t)=e^{-i t \delta \mu}\left[\alpha_{0}(x)+\delta \alpha(x, t)\right]$ or equivalently

$$
\chi(x, t)=e^{-i t \sigma_{3} \delta \mu}\left[\chi_{0}(x)+\delta \chi(x, t)\right],
$$

one obtains the collective Bogoliubov excitations modes and linear response frequencies of Eq. (79).

$$
\begin{aligned}
i \partial_{t} \delta \chi & =\Sigma^{\mathrm{B}}(x) \delta \chi(x, t)-\delta \mu P, \\
\Sigma_{\mathcal{N}}^{\mathrm{B}}(x) & =\frac{-\partial_{x}^{2}+x^{2}}{2}+2 g\left|\alpha_{0}(x)\right|^{2}-\mu, \\
\Sigma_{\mathcal{A}}^{\mathrm{B}}(x) & =g \alpha_{0}(x)^{2},
\end{aligned}
$$

The energy spectrum for the linear response matrix $\Sigma^{\mathrm{B}}$ must be gap-less $\left(E_{0}=0\right)$ as the GP-Eq. (79) is $U(1)$ invariant under a global phase change of $\alpha_{0}$. In other words, there exists a degenerate manifold of ground state solutions and it requires no energy to transform one of them into another. The infinitesimal generator of this phase rotation is the zero-mode $P=\left(\alpha_{0},-\alpha_{0}^{*}\right)^{\top}$ and it can be verified easily that $\Sigma^{\mathrm{B}} P=0$.

The initial perturbation $\delta \chi$ can also induce a small change in the particle number $\delta N=P^{\dagger} \sigma_{3} \delta \chi$. In turn, this leads to a small modifications of the chemical potential $\mu(N+\delta N)=$ $\mu(N)+\delta \mu$ at which the perturbed system is evolving globally. By taking this into account in the ansatz for the perturbation analysis in Eq. (81), one is able to cope with the secular terms that arise otherwise in a gap-less linear response analysis

$$
\begin{aligned}
\delta \chi(t) & =e^{-i t \Sigma^{\mathrm{B}}} \delta \chi(x, 0)+i t \delta \mu P \\
& =i Q \delta N-i P \delta \Phi+W^{\prime} e^{-i t E^{\prime}} \sigma_{3}^{(n-1)} \delta \chi^{\prime},
\end{aligned}
$$

where we have used the completeness relation of Eq. (43) and defined Bogoliubov amplitudes as $\delta \Phi=Q^{\dagger} \sigma_{3} \delta \chi$, as well as $\delta \chi^{\prime}=W^{\prime \dagger} \sigma_{3} \delta \chi$. The residual energy shift of the chemical potential is proportional to the inverse of the inertial mass of the collective ground state mode $\delta \mu=\delta N / M$. Within the $\mathrm{TF}$ approximation, one finds for the mass parameter $M_{\mathrm{TF}}=$ $(12 g N)^{1 / 3} / g$.

In considering the similarities of the collective Bogoliubov excitations Eq. (82) and the quasi-particle modes of the generalized self-energy matrix of Eq. (72), it is of utmost importance not to confuse their different physical meaning. Quasiparticle modes address the questions of excitations of the quantum fluctuations above a static mean-field without considering the back-action. Consequently, they do not have to be gap-less nor satisfy a Kohn theorem [74], but they are just a convenient basis to describe the quantum vacuum or the thermal excitations thereof. Thus, they should not be considered as better or worse approximation of each other. Only the linear response analysis of the coupled system of Eqs. (71, 72) will be comparable to the collective Bogoliubov excitations of Eq. (82). It is straight forward to verify that the coupled system of Eqs. $(71,72)$ are $U(1)$ invariant, thus gap-less $\left(E_{0}=0\right)$. Moreover, the collective center of mass oscillation also decouples from the internal excitations and it evolves exactly with the harmonic oscillator frequency of $E_{1}=1$.

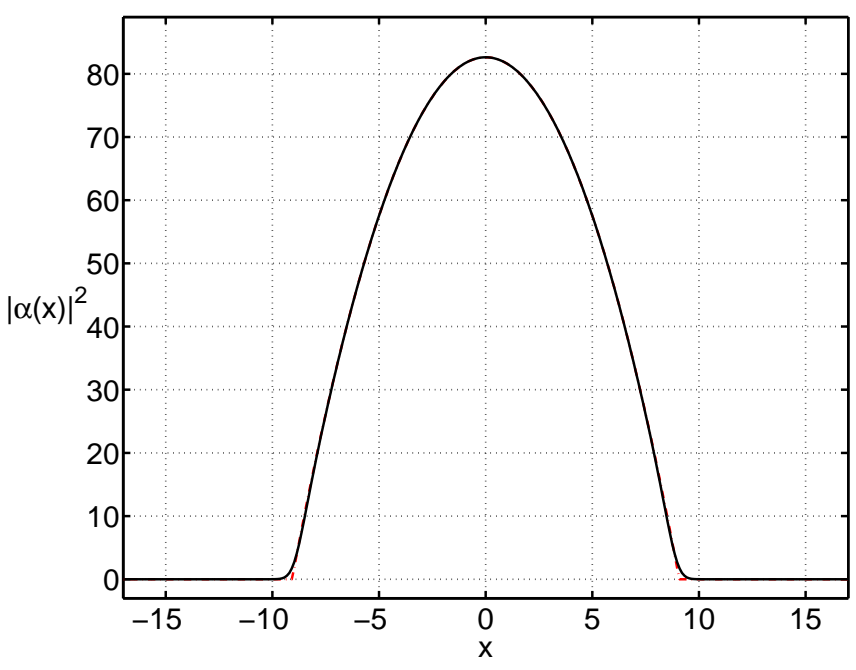

Figure 2: The condensate density $|\alpha(x)|^{2}$ as a function of the position $x$ in units of the harmonic oscillator length for a particle number of $N=10^{3}$. Only in the proximity of the Thomas-Fermi radius $x_{\mathrm{TF}}=9.07$ the exact numerical solution (solid line) is distinguishable from the Thomas-Fermi approximation (dashed doted line). The chemical potential energy in h.o. units $\mu=41.205$ is also well approximated by $\mu_{\mathrm{TF}}=41.198$.
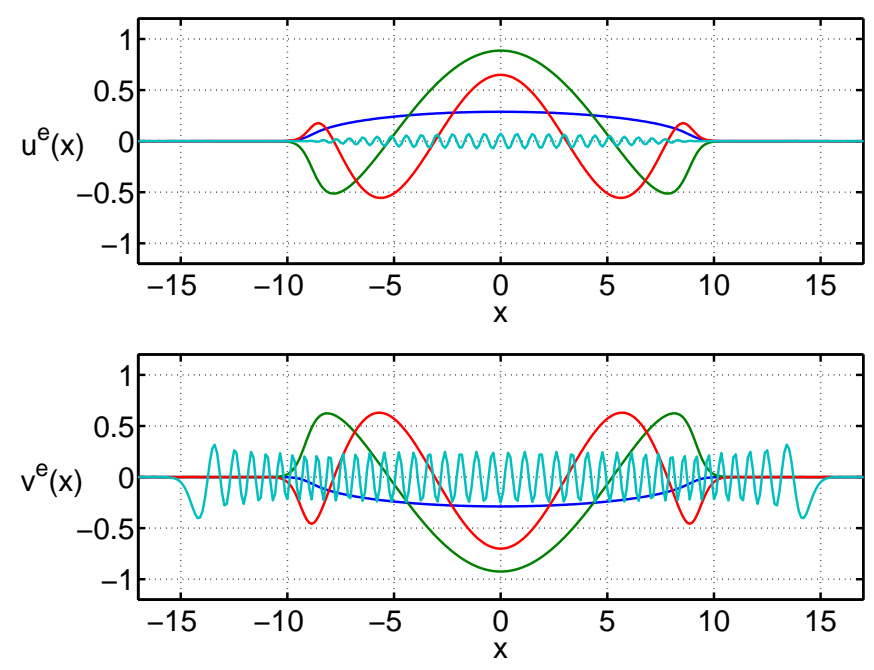

Figure 3: The normalized, even spatial components of the negative energy Bogoliubov excitation mode $W\left(E_{n}<0\right)=\left[u_{e}, v_{e}^{*}\right]^{\top}$ as a function of the position $x$ in units of the harmonic oscillator length. In particular, we show the three low energy modes $E_{n=(0,2,4)}=(0,-1.732,-3.167)$ and an arbitrarily chosen higher energetic mode $E_{80}=-63.845$ for comparison.

\section{Static mean-field and collective Bogoliubov excitations for $10^{3}$ particles}

In Figs. 3 and 4, we depict a few selected collective excitation modes of the GP-equation corresponding to the static solution with $N=10^{3}$ particles shown in Fig. 2. The reflexion symmetry of the harmonic trap is carried over to the mean- 

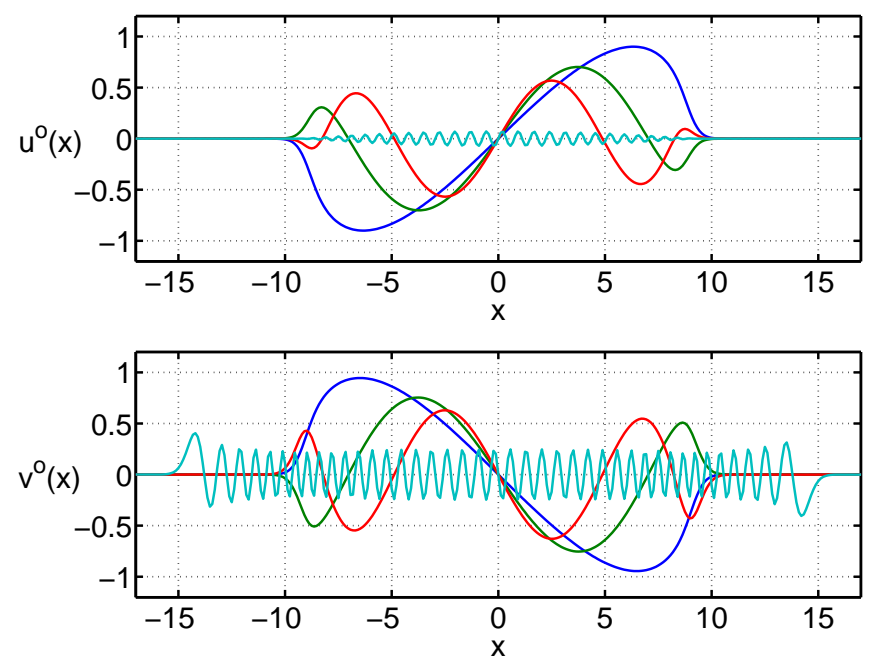

Figure 4: The normalized, odd spatial components of the negative energy Bogoliubov excitation mode $W\left(E_{n}<0\right)=\left[u_{o}, v_{o}^{*}\right]^{\top}$ as a function of the position $x$. Depicted are the three low energy modes $E_{n=(1,3,5)}=(-1,-2.451,-3.882)$ and an arbitrarily chosen higher energetic mode $E_{81}=-64.723$ for comparison.

field state. Consequently, one can also classify the collective Bogoliubov modes according to even and odd parity modes. Conventionally, one introduces also hole $u(E<0)$ and particle $v(E<0)$ amplitudes as components of $W\left(x, E_{n}<0\right)=$ $\left[u(x, E), v^{*}(x, E)\right]^{\top}$. The particle character of $v(E<0)$ becomes visible in the higher energy excitation modes $E_{80,81}$ where only the particle-like amplitudes reach far outside the spatial extend of the condensate and oscillate between the classical turning points of fictitious particles with energy $|E|$. In contrast, hole-like excitations always remain localized on the site of the condensate wave-function and their amplitudes decrease with decreasing excitation energy.

\section{Chemical potential and collective Bogoliubov excitation energies for a full range of particle numbers}

The general character of the solutions depends parametrically on the particle number. In Fig. 5, we have plotted the chemical potential $\mu(N)$ as well as the collective Bogoliubov energies $E(N)$ as a function of the particle number $N$. The double logarithmic representation of the chemical potential shows clearly a linear slope, hence reproduces the power law dependence that is found within the Thomas-Fermi approximation $\mu_{\mathrm{TF}}(N)=(3 g N / 2)^{2 / 3} / 2$. The collective excitation frequencies are identical to the harmonic oscillator spectrum for small particle numbers. However with increasing particle number, the energy density of states visibly increases as the level spacing is reduced. By focusing on the two lowest energy excitations, i.e., the Goldstone mode $E_{0}=0$ and Kohn mode $E_{1}=1$, one finds that those fundamental symmetry generating modes are unaffected by the interactions.
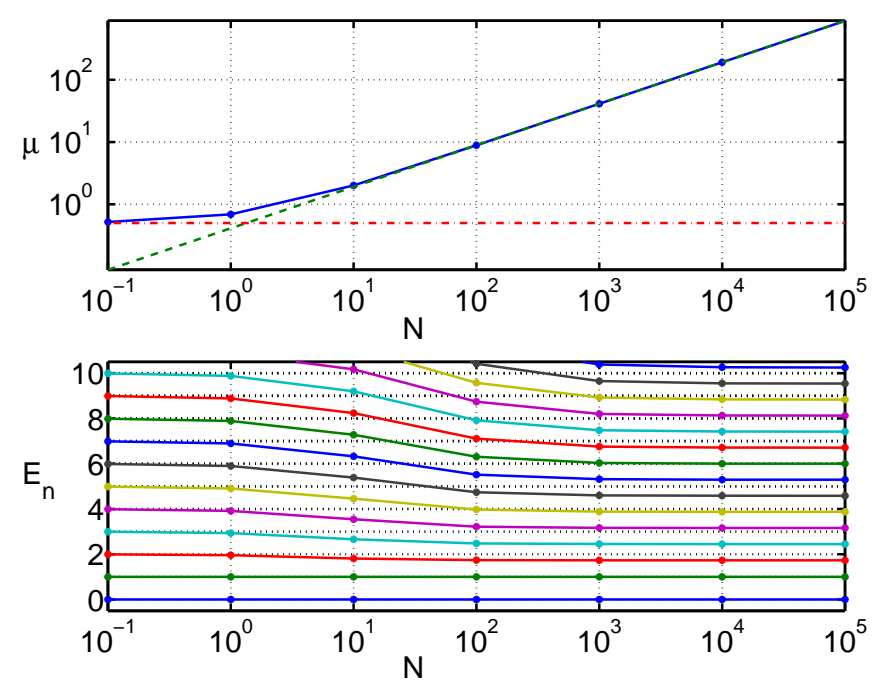

Figure 5: The upper part shows the chemical potential $\mu(N)$ of the GP equation as a function of particle number (solid line). For comparison, we present also the Thomas Fermi approximation $\mu_{\mathrm{TF}}(N)$ (dashed line) and the harmonic oscillator ground state energy of $1 / 2$ (dashes doted line). In the lower part, the collective Bogoliubov energies $E$ are shown versus particle number. All units are given in h.o. energies.

\section{Ground state solutions of the self-consistent Hartree-Fock-Bogoliubov equations}

In this section, we will discuss the self-consistent solution of the stationary Eqs. (71) and (72), i.e.,

$$
0=\Pi \chi, \quad 0=\Sigma G-G \Sigma^{\dagger} .
$$

In particular, we are interested in the lowest energy configuration of the system. Thus, no quasi-particle modes shall be occupied and according to Eq. (21), we construct the quantum vacuum only from the negative energy states $W_{-}=W_{E<0}$ with $\operatorname{dim}\left[W_{-}\right]=2 n \times n$

$$
G=W_{-} W_{-}^{\dagger} .
$$

With an iterative procedure, one can solve for the meanfield amplitude $\alpha_{0}$ assuming static $\tilde{f}$ and $\widetilde{m}$. In a second step, one has to find the quasi-particle modes and construct a density matrix from it. This procedure has to be continued until convergence is reached. We have computed the self-consistent solutions for a range of particle numbers $N=$ $\left(10^{0}, 10^{1}, 10^{2}, 10^{3}, 10^{4}, 10^{5}\right)$. For a particle number $N<1$, this extended mean-field theory is neither physically meaningful, nor did it lead to converging solutions any longer.

\section{Static mean-field and quasi-particle modes for $10^{3}$ particles}

For a particle number $N=10^{3}$, we show in Fig. 6 the mean-field density as a function of position. Due to a 


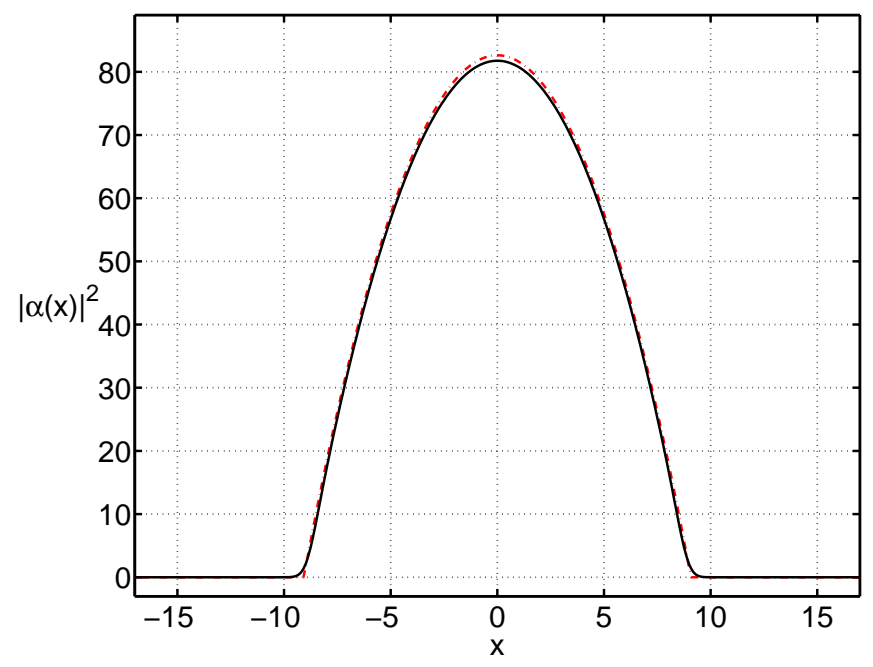

Figure 6: The self-consistent mean-field density $|\alpha(x)|^{2}$ (solid line) and Thomas-Fermi approximation (dashed doted line) as a function of the position $x$ in units of the h.o. length.
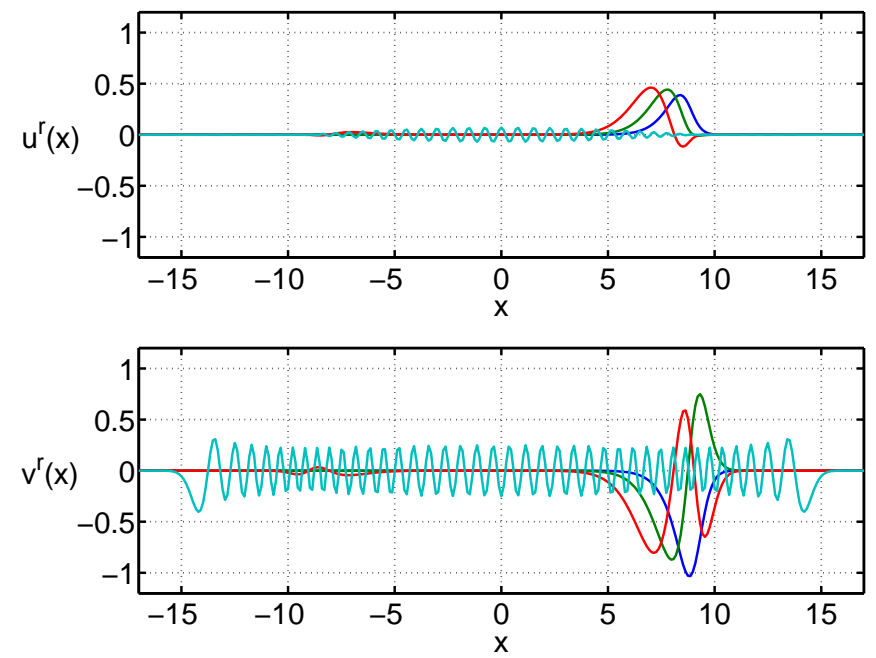

Figure 7: The normalized, spatial components of the negative energy Bogoliubov excitation mode $W\left(E_{n}<0\right)=\left[u_{r}, v_{r}^{*}\right]^{\top}$ as a function of the position $x$ in units of the harmonic oscillator length. In particular, we show the three low energy modes $E_{n=(0,2,4)}=$ $(-3.356,-6.421,-8.636)$ and an arbitrarily chosen higher energetic mode $E_{80}=-64.819$ for comparison. The low energy modes are localized on the right side of the condensate and almost degenerate to the modes localized on the other side depicted in Fig. 8.

repartitioning of particles between condensate and the noncondensed fraction, there are now fewer particles in the meanfield component. Thus, the difference to the Thomas-Fermi approximation is more visible than in Fig. 2. For a total particle number of $N=10^{3}=N^{(c)}+\tilde{N}$, we find a $\left(N^{(c)}, \tilde{N}\right)=$ $(984.83,15.17)$ and chemical potential of $\mu=40.61$ in h.o. energy units.

The quasi-particle modes are depicted in Figs. 7 and 8. While the collective Bogoliubov modes of Figs. 3 and 4 can be
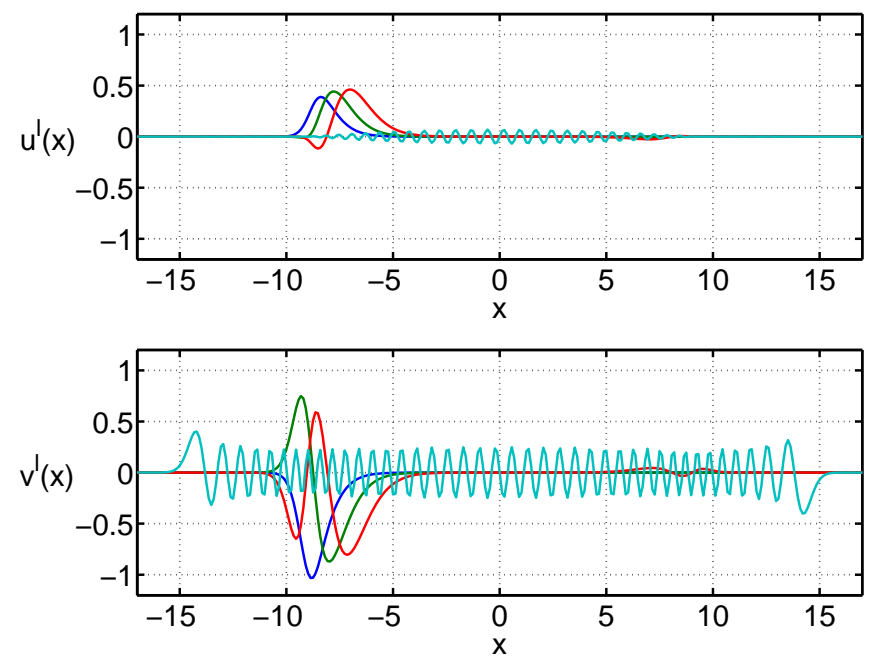

Figure 8: The normalized, spatial components of the negative energy Bogoliubov excitation mode $W\left(E_{n}<0\right)=\left[u_{l}, v_{l}^{*}\right]^{\top}$ as a function of the position $x$. Depicted are the three low energy modes $E_{n=(1,3,5)}=(-3.3556,-6.421,-8.636)$ and an arbitrarily chosen higher energetic mode $E_{81}=-65.689$ for comparison. The low energy modes are localized on the left side of the condensate.

characterized with a definite even or odd parity, this is seemingly not the case here. The low energy quasi-particle modes are localized on left and right sides of the condensate and have more of the character of the single particle Hartree-Fock excitations discussed in Eqs. (32) and (49), where the potential energy $V_{\mathrm{ho}}+2 \mathcal{U}_{f^{(c)}}-\mu$ has a double minimum at $\pm x_{\mathrm{TF}}$. However, one has to take into account also that the low lying modes are energetically degenerate and that the higher energy excitations do exhibit a definite parity. Thus, one could construct quasi-particle modes with definite parity by symmetrizing or anti-symmetrizing them. We have deliberately chosen not do so in order to permit the occurrence of a reflexion symmetry breaking. For example, spatial symmetry breaking is observed in deformed nuclei [58] or the mixing of two component Bose gases [75]. Nevertheless, we did not find such a symmetry breaking behavior here.

\section{Chemical potential and quasi-particle energies for a full range of particle numbers}

In Fig. 9, we present the chemical potential $\mu(N)$ as well as the quasi-particle energies $E$ as function of the total particle number $N$. One can see very clearly the energy gap $E_{0} \neq 0$ and the absence of the Kohn mode $E_{1} \neq 1$, which should occurs only for the collective excitations of system as whole. Moreover, one can observe how the low lying quasiparticle modes begin to coalesce with gradually increasing particle number. 

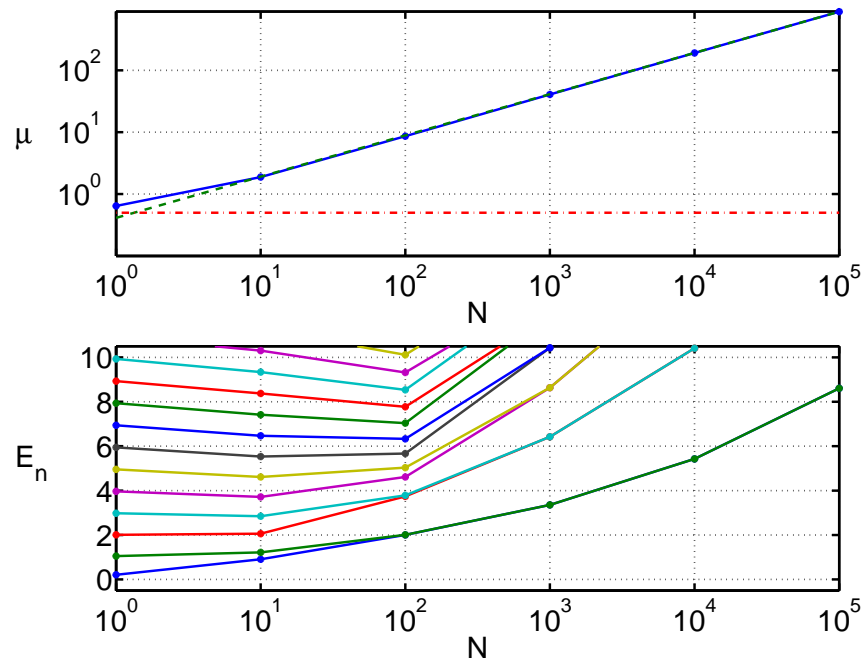

Figure 9: Upper part: the chemical potential $\mu(N)$ (solid line) of the SCHFB equations, the TF approximation $\mu_{\mathrm{TF}}$ (dashed line) and the h.o. ground state energy $1 / 2$ (dashed dotted line) versus particle number $N$. Lower part: quasi-particle energy spectrum $E(N)$ versus particle number $N$.

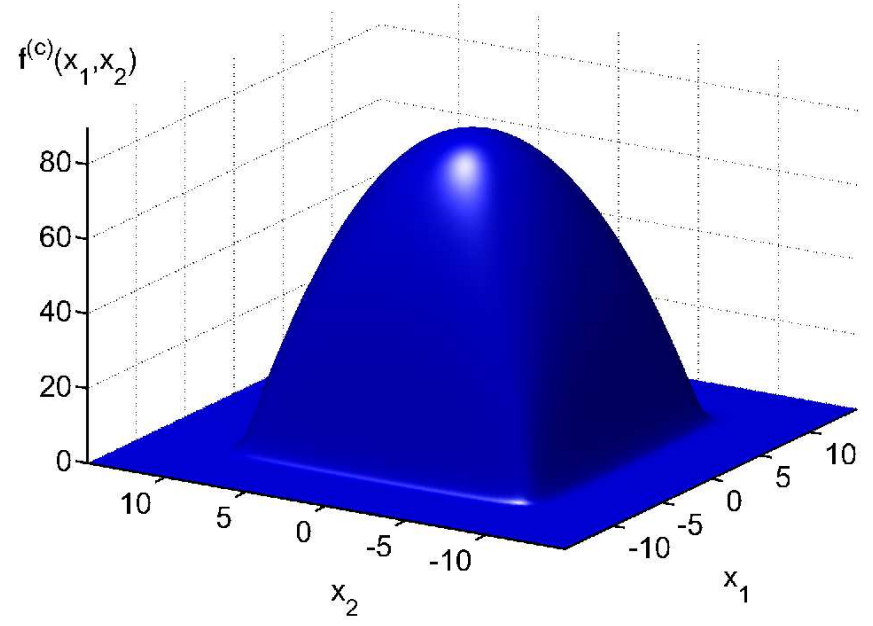

Figure 10: Coherent contribution of the single particle density matrix $f^{(c)}\left(x_{1}, x_{2}\right)$ as a function of the spatial coordinates for a particle number of $N=10^{3}$. The coherence extends in the diagonal as well as in off-diagonal directions up to the TF radius. For a real valued mean-field $\alpha(x)$, the coherent contribution of the pairing field $m^{(c)}\left(x_{1}, x_{2}\right)$ is also represented by this figure.

\section{The single particle density matrix and the pairing field}

The mean-field amplitude $\alpha(x)$, the total single particle density $f\left(x_{1}, x_{2}\right)=f^{(c)}\left(x_{1}, x_{2}\right)+\tilde{f}\left(x_{1}, x_{2}\right)$, as well as the pairing field $\widetilde{m}\left(x_{1}, x_{2}\right)$ have been the central concepts of the present analysis. Thus, we will present in the following pictures instances of their spatial representation for a particle number of $N=10^{3}$. This gives a good qualitative impression

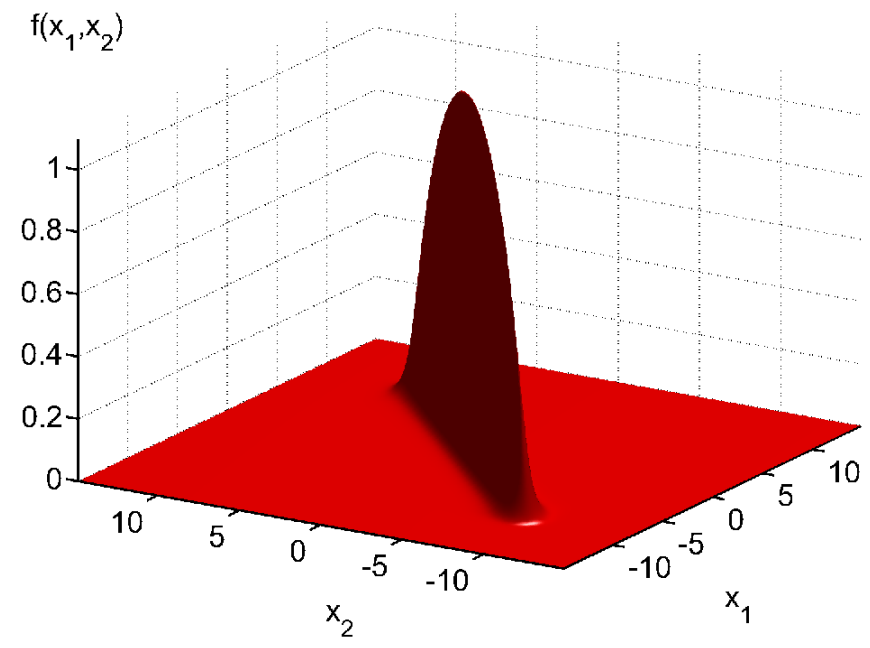

Figure 11: Non-condensate density matrix $\tilde{f}\left(x_{1}, x_{2}\right)$ as a function of the spatial coordinates for a particle number of $N=10^{3}$.

of the universal features of the ground state. Results obtained for different particle numbers are similar in appearance and we will discuss the quantitative differences next.

\section{Spatial representation for $10^{3}$ particles}

In Fig. 10, we show the coherent contribution to single particle density matrix $f^{(c)}\left(x_{1}, x_{2}\right)=\alpha^{*}\left(x_{2}\right) \alpha\left(x_{1}\right)$. As it is constructed from the order parameter $\alpha(x)$, it has the full ODLRO [20, 21], which extends over the complete system size. For the one-dimensional trap that we consider in here, the Hamiltonian operator is real valued, thus the ground state solution of the mean-field $\alpha(x)$ is a purely real quantity, too. Consequently, $m^{(c)}\left(x_{1}, x_{2}\right)=\alpha\left(x_{2}\right) \alpha\left(x_{1}\right)$ is identical to $f^{(c)}\left(x_{1}, x_{2}\right)$ as depicted in Fig. 10.

In contrast to the full ODLRO which is found in the meanfield component, one finds that the non-condensate density $\tilde{f}\left(x_{1}, x_{2}\right)$ is predominantly localized along the coordinate diagonal and exhibits spatial variation only due to the external confinement with a trapping potential. In the off-diagonal direction this order parameter has only a very short range which is determined by the binary interaction. This is depicted in Fig. 11.

Interestingly, one finds also for the pairing field $\widetilde{m}\left(x_{1}, x_{2}\right)$ a very similar spatial behavior. However, while a single particle interpretation is sufficient to understand the behavior of the normal density matrix, it is necessary to use two-particle physics in the pairing field of Fig. 12. The strong negative correlation along the diagonal show that there is a reduced likelihood of finding two particles at the same location. Again, this likelihood is modulated by the density of particles in trap. However, the degree of anti-correlation drops off quickly with increasing distance between the positions at which particles are extracted. 


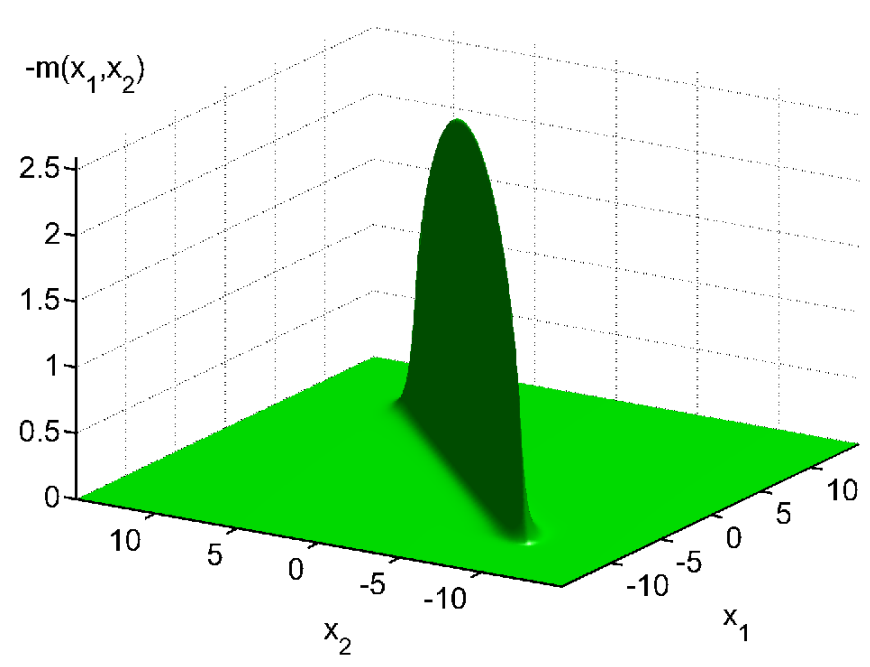

Figure 12: The negative pairing field $-\widetilde{m}\left(x_{1}, x_{2}\right)$ as a function of the spatial coordinates for a particle number of $N=10^{3}$.

\section{Diagonal and off-diagonal elements of $\tilde{f}$ and $\tilde{m}$ for a full range of particle numbers}

In this section, we have compiled the quantitative results for the spatial variation of the diagonal $\tilde{f}(x, x), \widetilde{m}(x, x)$ and the off-diagonal $\tilde{f}(x,-x), \widetilde{m}(x,-x)$ elements of the normal density matrix and the pairing field, respectively, for a full range of particle numbers $N=\left(10^{0}, \ldots, 10^{5}\right)$. The double logarithmic representation used in Figs. 13 and 14 reveals clearly that there is a separation of the bulk physics in the center of the trap and the physics dominated by the boundary at the rim of the condensate. We have verified this separation of scales by turning of the trap potential. In this case, one recovers the homogeneous limit. In order to make a quantitative comparison with the trapped system, we have chosen a homogeneous mean-field density $n=N / 2 L=82.62$ that matches the mean-field density at the center of the trapped gas for a particle number of $N=10^{3}$. This leads to very similar chemical potentials for the homogeneous system of $\mu_{\mathrm{hom}}=40.47$ and for the trapped gas of $\mu_{\text {trap }}=40.61$, respectively. The dashed dotted lines in Figs. 13 and 14, do represent the homogeneous results and compare very well with the trapped gas for $N=10^{3}$. This proves that the local density approximation (LDA) yields a good approximation for the transverse correlation length. A detailed comparison of the critical exponents and their dependence on temperature is currently under investigation.

\section{E. Effective coupling constants}

In Sec. II F, we have discussed formally the renormalization of the binary interaction to an effective T-matrix, which takes place in any self-consistent calculation. In particular, we upgraded the anomalous potential of Eq. (53) and related it to an anomalous many-body T-matrix. As we have calculated
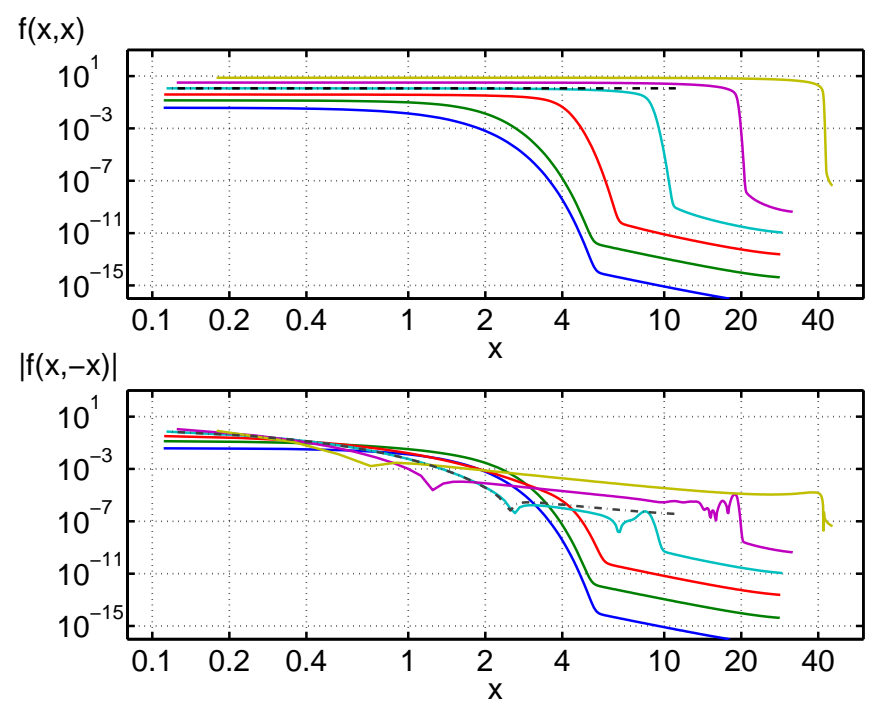

Figure 13: Diagonal $\tilde{f}(x, x)$ and off-diagonal $|\tilde{f}(x,-x)|$ elements of the normal density matrix versus distance $x$ for a full range of particle numbers $N=\left(10^{0}, \ldots, 10^{5}\right)$. The individual results can be identified easily by the spatial extension that grows proportional with the particle number. The dashed-dotted line shows the result for a homogeneous gas corresponding to a trapped gas with $N=10^{3}$ particles.

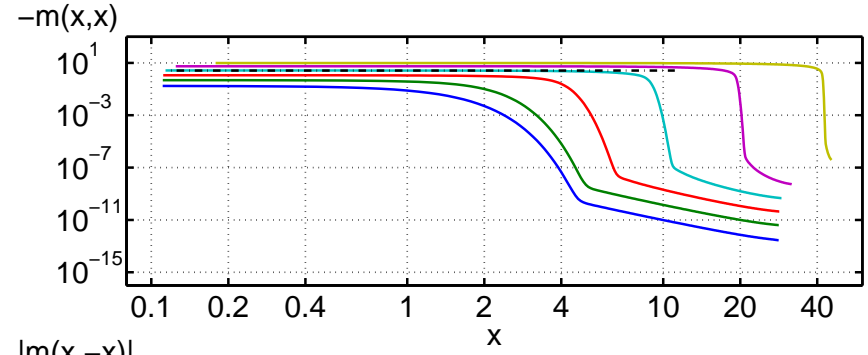

$|\mathrm{m}(\mathrm{x},-\mathrm{x})|$

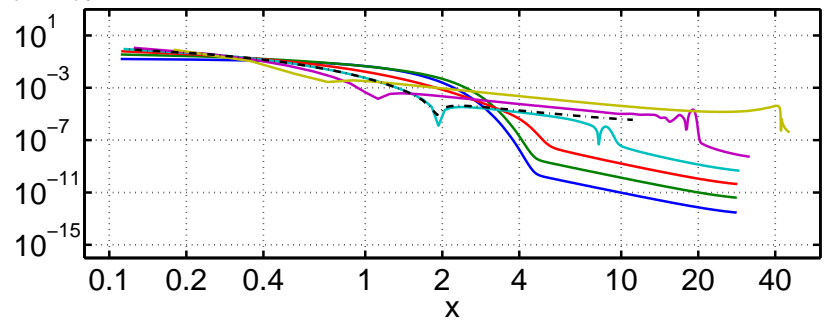

Figure 14: Diagonal $-\widetilde{m}(x, x)$ and off-diagonal $|\widetilde{m}(x,-x)|$ elements of the pairing field versus distance $x$ as in Fig. 13.

the SCHFB equations in here, we can now revisit this question and find out what the effective renormalized coupling constant is

$$
\begin{aligned}
T_{\mathcal{A}}(2 \mu, x) m^{(c)}(x, x) & =g\left[m^{(c)}(x, x)+\widetilde{m}(x, x)\right], \\
T_{\mathcal{N}}(2 \mu, x) f^{(c)}(x, x) & =2 g\left[f^{(c)}(x, x)+\tilde{f}(x, x)\right] .
\end{aligned}
$$

From the numerical results of Fig. 15, we can draw three conclusions: a) the effective coupling is position (or momentum) 

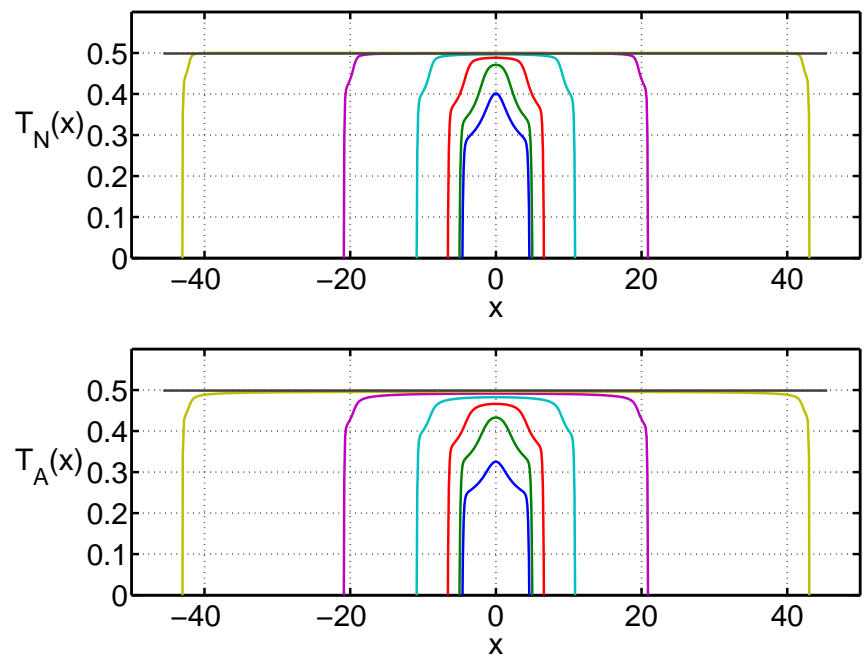

Figure 15: The effective coupling constants $T_{\mathcal{N}}(2 \mu, x)$ and $T_{\mathcal{A}}(2 \mu, x)$ as a function of position. Each subplot contains six individual lines corresponding to the full range of particle numbers $N=\left(10^{0}, \ldots, 10^{5}\right)$ and a flat line, which the represents the bare coupling constant $g=0.4986$. The individual results can be identified easily by their spatial extension, which increases proportional to the $\mathrm{TF}$ radius or particle number, respectively.

dependent and decreases rapidly outside of the range of the condensate; b) the effective coupling constant is in general less than the bare interaction constant, which may be understood in terms of a second order perturbation theory; c) the effective coupling constant at the center of the trap becomes gradually less for smaller particle numbers.

\section{F. First and second order correlations functions}

\section{Spatial representation for $10^{3}$ particles}

Quantum fluctuations around the classical mean-field amplitude are the central topic of this article. In the previous sections, we have examined the single particle density $f_{x}=f^{(c)}+\tilde{f}$, which is directly an observable quantity and the pair correlation function $\widetilde{m}\left(x_{1}, x_{2}\right)$, which is not. The questions of how to quantify and to measure quantum correlations has always been a central theme for any quantized field theory, whether in condensed matter physics $[20,21,32]$ or in quantum optics [76, 77].

In essence, first order coherence is measured by the correlation function

$$
\begin{aligned}
g^{(1)}\left(x_{1}, x_{2}\right) & =\frac{\left\langle\hat{a}_{x_{2}}^{\dagger} \hat{a}_{x_{1}}\right\rangle}{\sqrt{n\left(x_{1}\right) n\left(x_{2}\right)}} \\
& =\frac{f^{(c)}\left(x_{1}, x_{2}\right)+\tilde{f}\left(x_{1}, x_{2}\right)}{\sqrt{n\left(x_{1}\right) n\left(x_{2}\right)}},
\end{aligned}
$$

where $n(x)=f^{(c)}(x, x)+\tilde{f}(x, x)$ denotes the total density. Primarily, it is sensitive only to spatial phase correlations or,

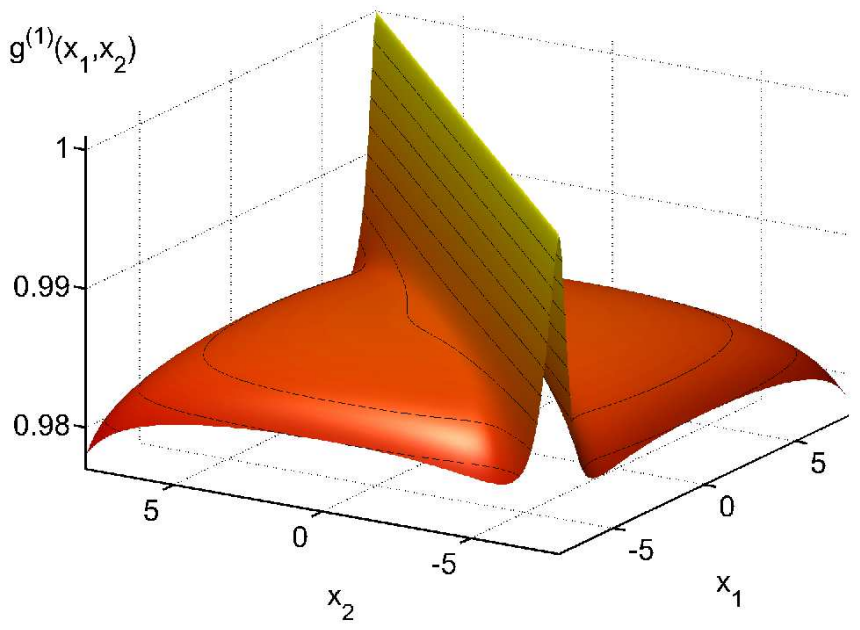

Figure 16: First order correlation function $g^{(1)}\left(x_{1}, x_{2}\right)$ versus spatial position for $10^{3}$ particles.

in other words, off-diagonal order. This can bee seen easily by disregarding the quantum depletion $\tilde{f}$ for the moment. In the case of a mean-field $\alpha(x)=|\alpha(x)| e^{-i \Phi(x)}$, it is only proportional to the phase gradients $\Phi(x)=\Phi(0)+\delta \Phi(x)$. Moreover, if $\alpha(x)$ is a static ground state without a phase gradient (irrotational), one finds the definition of full coherence, i. e., $g^{(1)}\left(x_{1}, x_{2}\right)=1$.

In Fig. 16, we have evaluated the first order correlation function for a trapped gas with $N=10^{3}$ particles. By definition, it must be exactly 1 along the diagonal. As expected, there is only a very weak influence of the quantum fluctuations noticeable, since $f^{(c)}\left(x_{1}, x_{2}\right) \gg \tilde{f}\left(x_{1}, x_{2}\right)$, which can be seen explicitly in Fig. 6 and Fig. 11. Consequently, one finds that first order coherence is not a very sensitive probe for the quantum aspects of a field. For example, sending a classical optical field through an semi-opaque or noisy medium immediately leads to a reduction coherence, which is as such a purely classical phenomenon.

Actually, a sensitive probe for the quantum nature of a field is the second order correlation function $g^{(2)}$. If $g^{(1)}$ basically responds to uncertainty in the phase quadrature, then $g^{(2)}$ is affected by density fluctuations $\hat{a}_{x}^{\dagger} \hat{a}_{x}=n(x)+\delta \hat{n}(x)$. Intuitively speaking, this is the conjugate variable to the phase gradient. More succinctly speaking, this can be examined by studying a Heisenberg uncertainty product (see App. A) for all the field quadratures as in Eq. (46). Explicitly, it is defined as the normal ordered density-density correlation function:

$$
\begin{aligned}
& g^{(2)}\left(x_{1}, x_{2}\right)=\frac{\left\langle\hat{a}_{x_{1}}^{\dagger} \hat{a}_{x_{2}}^{\dagger} \hat{a}_{x_{2}} \hat{a}_{x_{1}}\right\rangle}{n\left(x_{1}\right) n\left(x_{2}\right)}=1+\frac{1}{n\left(x_{1}\right) n\left(x_{2}\right)}\{ \\
& +2 \Re\left[f^{(c)}\left(x_{1}, x_{2}\right)^{*} \tilde{f}\left(x_{2}, x_{1}\right)+m^{(c)}\left(x_{1}, x_{2}\right)^{*} \widetilde{m}\left(x_{2}, x_{1}\right)\right] \\
& \left.+\tilde{f}\left(x_{1}, x_{2}\right) \tilde{f}\left(x_{2}, x_{1}\right)+\widetilde{m}\left(x_{1}, x_{2}\right)^{*} \widetilde{m}\left(x_{2}, x_{1}\right)\right\}
\end{aligned}
$$

which is shown in Fig. 17 for $10^{3}$ particles. While $g^{(2)}$ it is mostly equal to 1 , it can be seen clearly that along the diagonal 


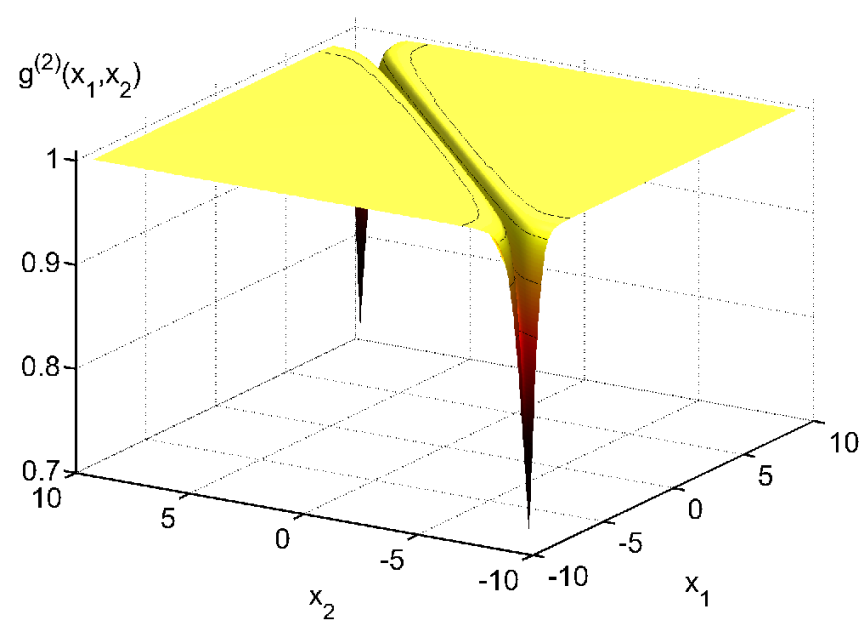

Figure 17: Second order correlation function $g^{(2)}\left(x_{1}, x_{2}\right)$ versus spatial position for $10^{3}$ particles.

$g^{(2)}(x, x)<1$. This is a unique signature a non-classical state of the quantum field with a sub-poisonian statistic of number fluctuations or, in other words, a number squeezed state [34]. The suppression of number fluctuations has its origin the binary interaction potential of Eq. (1). By reducing the local density fluctuations, one can remove more energy than what is regained by the kinetic energy due to the increased phase uncertainty. At its extreme this is also the physical mechanism that leads to the Mott phase transition [11-13, 78] where on-site interactions compete with nearest neighbor tunneling.

\section{Diagonal and off-diagonal elements of the first and second order correlation function for a full range of particle numbers}

Finally, we summarize the results for the first and second order correlation function in Figs. 18 and 19 for the full range of particle numbers $N=\left(10^{0}, \ldots, 10^{5}\right)$. As before, we have also computed the result for a homogeneous gas in the local density approximation, which corresponds to a trapped gas of $10^{3}$ particles. The insets in the pictures magnify both of those curves in the central region where they do agree very well. However, at the rim of the condensate the trapped gas does exhibit features that are absent in the LDA.

It is physically most important to see in Figs. 19 that the suppression density fluctuations becomes stronger for smaller particle numbers. At its limit, this anti-correlation of tow bosonic particles leads to an effective fermionization and is the hall mark of the Tonks-Girardeau regime [18, 37, 50]. If this argument is applied to the situation of an inhomogeneous trapped gas, then this means that the number squeezing is larger at the rim than in the center of the cloud.

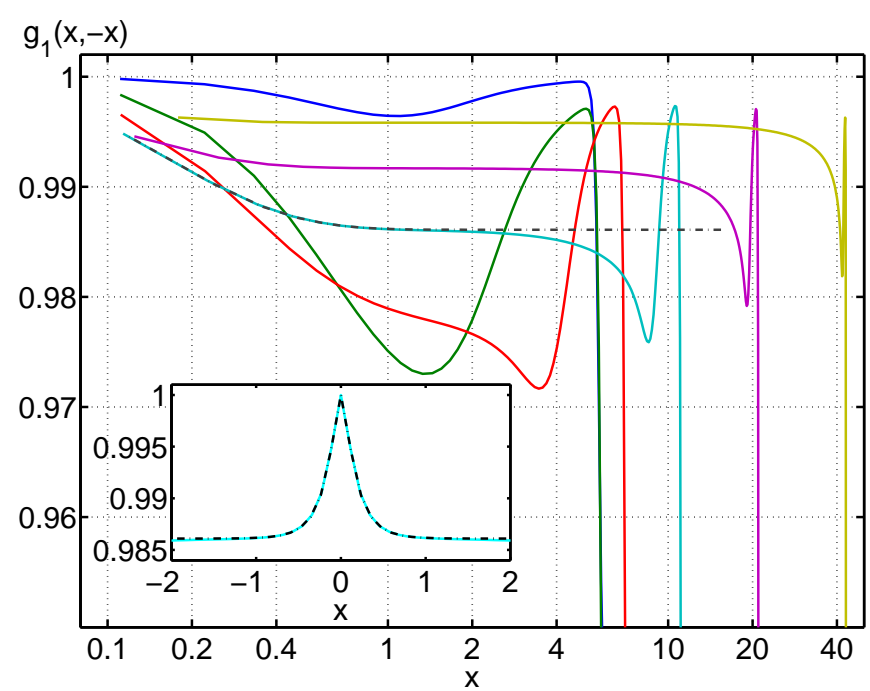

Figure 18: Off-diagonal elements of the first order correlation function $g^{(1)}(x,-x)$ versus spatial position. Each subplot contains six individual lines corresponding to the full range of particle numbers $N=\left(10^{0}, \ldots, 10^{5}\right)$ and a dashed dotted line, which represents the homogeneous gas result. The individual results can be identified easily by their spatial extension, which increases proportional to the particle number.

$g_{2}(x, x)$
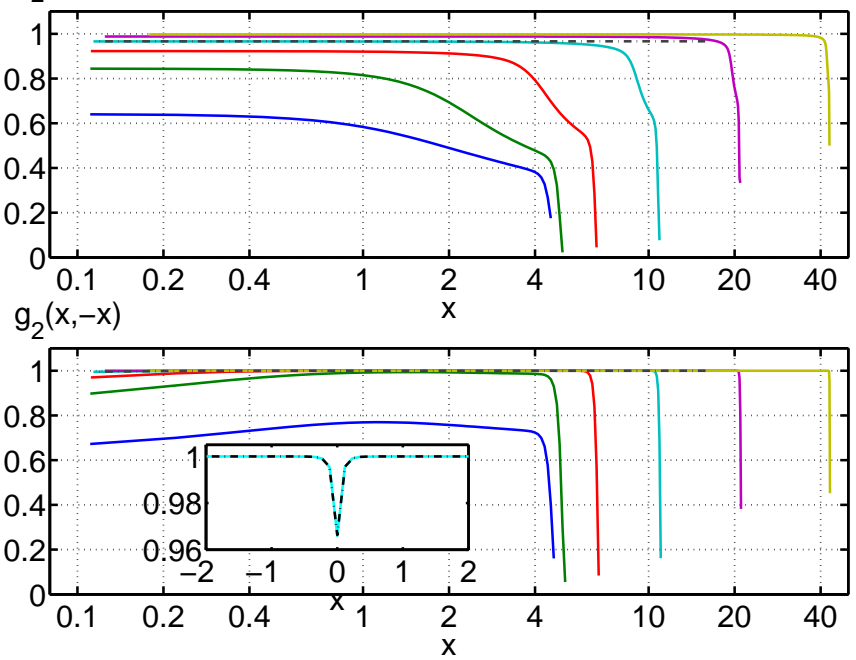

Figure 19: Diagonal $g^{(2)}(x, x)$ and off-diagonal $g^{(2)}(x,-x)$ elements of the second order correlation function versus spatial position. Parameters and legend as in Fig. (18).

\section{CONCLUSIONS AND OUTLOOK}

In this article, we have given a basic overview of the premises and concepts of a number-symmetry broken nonequilibrium kinetic theory of a trapped bosonic gas. By extending the successful mean-field concept of the GrossPitaevskii equation with the effects of non-local, two particle 
quantum correlations, one obtains a renormalized binary interaction T-matrix and allows for the dynamic establishment of non-classical many-particle quantum correlations. At very low temperatures, we can disregard the equilibrating effects of elastic two-particle collisions, in contrast to previous work [27, 28, 71]. In this limit, we have proven that the important physical constants of motion, like particle number, energy or the entropy are conserved. Obviously, the inclusion of collision processes is desirable from a fundamental point of view, as this will break the micro-reversibility and lead to a thermal relaxation towards the most probable distribution as dictated by thermodynamics. In preliminary study [31], we have already studied the effects of collisions and the consequences of various approximations in the absence of a mean-field in a model system, but further work is necessary.

In the second section of the paper, we have focused on the specific properties of the zero-temperature ground state correlations of a trapped, quasi-one dimensional bosonic gas. This maximizes the role played by quantum fluctuations, due to the dimensional reduction of the available phase-space volume. With a fully self-consistent numerical calculation, we have evaluated, the mean-field amplitudes, the quantum depletion, the pairing field as well as the first and second order ground state quantum correlations for a full range of particle numbers $N=\left(10^{0}, \ldots, 10^{5}\right)$. Most interestingly, we do get a strong suppression of the density fluctuations or, in other words, an enhanced number squeezing with decreasing particle density. This generic feature is in general agreement with the predictions that are found with exactly solvable onedimensional models such as the Tonks-Girardeau gas or the Bose-Hubbard gas on an optical lattice. A detailed analytical comparison with those models [37-39] and the experimental results $[40,43]$ is currently work in progress. In conclusion, we find that the general non-equilibrium kinetic theory also reproduces the ground state correlations of a quasi onedimensional bosonic gas well.

\section{Acknowledgments}

R. W. acknowledges gratefully many stimulating discussions with J. Cooper and F. Sols as well as travel support from the ESF through the BEC2000 program.

\section{Appendix A: CAUCHY-SCHWARTZ INEQUALITY}

For a positive semi-definite density operator $\sigma$ and an arbitrary operator $\hat{L}$ it follows that the expectation value

$$
\left\langle\hat{L} \hat{L}^{\dagger}\right\rangle=\operatorname{Tr}\left\{\sigma \hat{L} \hat{L}^{\dagger}\right\} \geq 0
$$

is never negative. Consequently, the co-variance matrix $G$ of Eq. (14) must be positive semi-definite $u^{\dagger} G u \geq 0$, as well. This can be easily seen, by considering a linear combination of two arbitrary operators $\hat{A}$ and $\hat{B}$, i. e. , $L=\alpha \hat{A}+\beta \hat{B}$. By minimizing the positive expression Eq. (A1), one obtains the
Cauchy-Schwartz inequality as

$$
\left\langle\hat{A} \hat{A}^{\dagger}\right\rangle\left\langle\hat{B} \hat{B}^{\dagger}\right\rangle \geq\left\langle\hat{B} \hat{A}^{\dagger}\right\rangle\left\langle\hat{A} \hat{B}^{\dagger}\right\rangle
$$

In particular, for the special choice of $\hat{A}=\delta \hat{a}_{1}$ and $\hat{B}=\delta \hat{a}_{2}^{\dagger}$, this implies that the magnitude of the anomalous fluctuations is limited by

$$
\left(1+\tilde{f}_{11}\right) \tilde{f}_{22} \geq\left|\widetilde{m}_{12}\right|^{2} .
$$

\section{Appendix B: PAULI MATRICES}

In this article, we use the following standard representation for the Pauli matrices:

$$
\sigma_{1}=\left(\begin{array}{ll}
0 & \mathbb{1} \\
\mathbb{1} & 0
\end{array}\right), \sigma_{2}=i\left(\begin{array}{cc}
0 & -\mathbb{1} \\
\mathbb{1} & 0
\end{array}\right), \sigma_{3}=\left(\begin{array}{cc}
\mathbb{1} & 0 \\
0 & -\mathbb{1}
\end{array}\right)
$$

They satisfy the ordinary commutation relation of an angular momentum operator $\left[\sigma_{1}, \sigma_{2}\right]=i \sigma_{3}$ and all cyclic permutations thereof. If $n$ is the dimension of the vector space, then $\sigma_{k}$ acts in a $2 n$-dimensional symplectic vector space [58].

\section{Appendix C: CANONICAL TRANSFORMATIONS}

A canonical transformation is an inhomogeneous linear combination of creation and destruction operators that preserves the commutation relation [58]. In particular, if $\hat{a}$ and $\hat{a}^{\dagger}$ denotes a pair of hermitian conjugated bosonic operators, such that

$$
\left[\hat{a}_{1}, \hat{a}_{2}^{\dagger}\right]=\delta_{1,2}
$$

then any affine linear transformation defines a new set of operators $b$ and $\bar{b}$ by

$$
\left(\begin{array}{c}
b \\
\bar{b}
\end{array}\right)=T\left(\begin{array}{c}
\hat{a} \\
\hat{a}^{\dagger}
\end{array}\right)+d
$$

In an $n$-dimensional vector space, $T$ represents a $2 n \times 2 n$ dimensional matrix and $d$ is a $2 n$ dimensional vector. Such a transformation is canonical if the new pair of operators also satisfies the commutation relation:

$$
\left[b_{1}, \bar{b}_{2}\right]=\delta_{1,2}
$$

More specifically, the transformation is unitary canonical if the new operators are hermitian conjugate pairs, i. e., $\bar{b}=b^{\dagger}$. By inserting Eq. (C2) into Eq. (C3), one finds that the transformation matrices are a representation of the symplectic group $S p(2 n)$ :

$$
T \sigma_{3} T^{\dagger}=\sigma_{3}
$$

In addition, it can be shown that $T^{*}=\sigma_{1} T \sigma_{1}$ and $T^{-1}=$ $\sigma_{3} T^{\dagger} \sigma_{3}$. 


\section{Appendix D: QUANTUM LIMIT FOR THE GROUND STATE CORRELATIONS}

The state of the interacting many-body system is described within the set of approximations by a mean-field amplitude $\alpha$ and a generalized $G$ matrix. At finite temperature or out of equilibrium, there is a finite occupation number of particles of positive energy excited states. However, in the lowest energy configuration all allocatable real particles occupy the meanfield amplitude and the generalized density matrix only holds the vacuum. Then, the following idem-potency relation holds for the density matrix

$$
G \sigma_{3} G+G=0
$$

This follows straight from Eq. (21) when $P_{+}=0$. Consequently, this gives a restriction for the components of the density matrix $\tilde{f}, \widetilde{m}$. In particular, one finds that the vacuum depletion is completely determined by the pairing field

$$
\begin{gathered}
\tilde{f}=\frac{1}{2}\left(\sqrt{\mathbb{1}+4 \tilde{m} \tilde{m}^{\dagger}}-\mathbb{1}\right), \\
\tilde{f} \tilde{m}=\tilde{m} \tilde{f}^{*} .
\end{gathered}
$$

It is important to note that this relation also holds out-ofequilibrium as long as thermalizing collisions can be disregarded.

\section{Appendix E: A GENERALIZED WICK'S THEOREM}

Gaußian fluctuations around a well defined mean value are the key assertion to apply Wick's theorem [56]. This is a set of rules to efficiently evaluate quantum averages for multiple operator products as

$$
\left\langle\hat{\psi}_{1} \hat{\psi}_{2} \ldots \hat{\psi}_{l}\right\rangle_{\left\{\alpha, \alpha^{*}, \tilde{f}, \tilde{m}\right\}}
$$

In this average, for example, the operator $\hat{\psi}_{1}$ represents either an operator $\hat{a}_{1}$ or $\hat{a}_{1}^{\dagger}$.

First, the displacement rule shifts any operator $\hat{\psi}_{1}$ by its cnumber expectation value $\psi_{1}$ which is either $\alpha_{1}$ or $\alpha_{1}^{*}$, and replaces the quantum average by an average that has zero mean values:

$$
\begin{aligned}
& \left\langle\hat{\psi}_{1} \hat{\psi}_{2} \ldots \hat{\psi}_{l}\right\rangle_{\left\{\alpha, \alpha^{*}, \tilde{f}, \tilde{m}\right\}}= \\
& \quad=\left\langle\left(\hat{\psi}_{1}+\psi_{1}\right)\left(\hat{\psi}_{2}+\psi_{2}\right) \ldots\left(\hat{\psi}_{n}+\psi_{l}\right)\right\rangle_{\{0,0, \tilde{f}, \tilde{m}\}}
\end{aligned}
$$

Second, after expanding the multiple products, one can discard all averages that involve an odd numbers of operators:

$$
\left\langle\hat{\psi}_{1} \hat{\psi}_{2} \ldots \hat{\psi}_{2 s+1}\right\rangle_{\{0,0, \tilde{f}, \tilde{m}\}}=0 .
$$

And third, for the remaining averages, one can use the Gaußian factorization rule:

$$
\begin{aligned}
& \left\langle\hat{\psi}_{1} \hat{\psi}_{2} \ldots \hat{\psi}_{2 s}\right\rangle_{\{0,0, \tilde{f}, \tilde{m}\}}= \\
& =\left\langle\hat{\psi}_{1} \hat{\psi}_{2}\right\rangle_{\{0,0, \tilde{f}, \tilde{m}\}}\left\langle\hat{\psi}_{3} \ldots \hat{\psi}_{2 s}\right\rangle_{\{0,0, \tilde{f}, \tilde{m}\}}+ \\
& +\left\langle\hat{\psi}_{1} \hat{\psi}_{3}\right\rangle_{\{0,0, \tilde{f}, \tilde{m}\}}\left\langle\hat{\psi}_{2} \hat{\psi}_{4} \ldots \hat{\psi}_{2 s}\right\rangle_{\{0,0, \tilde{f}, \tilde{m}\}}+ \\
& \quad \vdots \\
& +\left\langle\hat{\psi}_{1} \hat{\psi}_{2 s}\right\rangle_{\{0,0, \tilde{f}, \tilde{m}\}}\left\langle\hat{\psi}_{2} \ldots \hat{\psi}_{2 s-1}\right\rangle_{\{0,0, \tilde{f}, \tilde{m}\}} .
\end{aligned}
$$

By proceeding recursively, one has finally evaluated the complete multiple operator average Eq. (E1).
[1] F. Dalfovo, S. Giorgini, L. Pitaevskii, and S. Stringari, "Theory of trapped Bose-condensed gases," Rev. Mod. Phys. 71, 463 (1999).

[2] A. Leggett, "Bose-Einstein condensation in the alkali gases: Some fundamental concepts," Rev. Mod. Phys. 73, 307 (2001).

[3] K. Southwell, "Ultra cold matter," Nature 416, 205 (2002).

[4] C. Pethick and H. Smith, Bose-Einstein Condenstion in Dilute Gases (Cambridge University Press, 2002).

[5] M. H. Anderson and J. R. Ensher and M. R. Matthews and C. E. Wieman and E. A. Cornell, "Observation of Bose-Einstein Condesation in a Dilute Atomic Vapour," Science 269, 198 (1995).

[6] K. B. Davis, M.-O. Mewes, M. R. Andrews, N. J. van Druten, D. S. Durfee, D. M. Kurn, and W. Ketterle, "Bose-Einstein condensation in a gas of sodium atoms," Phys. Rev. Lett. 75, 3969 (1995).

[7] C. C. Bradley, C. A. Sackett, J. J. Tollett, and R. G. Hulet, "Evidence of Bose-Einstein Condensation in an Atomic Gas with Attractive Interactions," Phys. Rev. Lett. 75, 1687 (1995).

[8] J. Williams and M. Holland, "Preparing topological states of a Bose-Einstein condensate," Nature 401, 568 (1999).

[9] M. Matthews, B. Anderson, P. Haljan, D. Hall, C. Wieman, and E. Cornell, "Vortices in a Bose-Einstein Condensate," Phys. Rev. Lett. 83, 2498 (1999).

[10] J. Abo-Shaeer, C. Raman, J. Vogels, and W. Ketterle, "Observation of Vortex Lattices in Bose-Einstein Condensates," Science 292, 476 (2001).

[11] D. Jaksch, C. Bruder, J. Cirac, C. Gardiner, and P. Zoller, "Cold bosonic atoms in optical lattices," Phys. Rev. Lett. 81, 3108 (1998).

[12] M. Greiner, O. Mandel, T. Esslinger, T. Hänsch, and I. Bloch, "Quantum phase transitions from a superfluid to a Mott insulator in a gas of ultracold atoms," Nature 415, 39 (2002).

[13] M. Greiner, O. Mandel, T. W. Hänsch, and I. Bloch, "Collapse and revival of the matter wave field of a Bose-Einstein condensate," Nature 419, 51 (2002).

[14] C. Regal, C. Ticknor, J. Bohn, and D. Jin, "Creation of ultracold molecules from a Fermi gas of atoms," Nature 424, 47 (2003).

[15] M. Zwierlein, C. Stan, C. Schunck, S. Raupach, A. Kerman, and W. Ketterle, "Condensation of Pairs of Fermionic Atoms near a Feshbach Resonance,” Phys. Rev. Lett. 92, 120403 (2004).

[16] C. Regal, M. Greiner, and D. Jin, "Observation of Resonance Condensation of Fermionic Atom Pairs," Phys. Rev. Lett. 92, 040403 (2004). 
[17] C. Chin, M. Bartenstein, A. Altmeyer, S. Riedl, S. Jochim, J. H. Denschlag, and R. Grimm, "Observation of the Pairing Gap in a Strongly Interacting Fermi Gas,” Science 305, 1128 (2004).

[18] B. Paredes, A. Widera, V. Murg, O. Mandel, S. Fölling, I. Cirac, G. V. Shlyapnikov, T. W. Hänsch, and I. Bloch, "TonksGirardeau gas of ultracold atoms in an optical lattice," Nature 429, 277 (2004).

[19] K. Das, M. Girardeau, and E. Wright, "Crossover from One to Three Dimensions for a Gas of Hard-Core Bosons," Phys. Rev. Lett. 89, 110402 (2002), and references therein.

[20] O. Onsager and L. Penrose, "Bose-Einstein Condensation and Liquid Helium,” Phys. Rev 104, 576 (1956).

[21] C. Yang, "Concept of Off-Diagonal Long-Range Order and the Quantum Phases of Liquid He and of superconductors," Rev. Mod. Phys. 34, 694 (1962).

[22] S. Beliaev, "Energy-Spectrum of a Non-Ideal Bose Gas," JETP 34, 299 (1958).

[23] P. Hohenberg and P. Martin, "Microscopic Theory of Superfluid Helium,” Ann. Phys. 34, 291 (1965).

[24] E. Zaremba, T. Nikuni, and A. Griffin, "Dynamics of trapped bose gases at finite temperatures," J. Low. Temp. Phys. 116, 277 (1999).

[25] M. Rusch and K. Burnett, "Mean-field theory of excitations of trapped Bose condesates at finite temperatures," Phys. Rev. A 59, 3851 (1999).

[26] H. T. C. Stoof, "Coherent Versus Incoherent Dynamics During Bose-Einstein Condensation in Atomic Gases," J. Low Temp. Phys. 114, 11 (1999).

[27] R. Walser, J. Williams, J. Cooper, and M. Holland, "Quantum kinetic theory for a condensed bosonic gas," Phys. Rev. A 59, 3878 (1999).

[28] J. Wachter, R. Walser, J. Cooper, and M. Holland, "Equivalent kinetic theories of Bose-Einstein condensation," Phys. Rev. A 64, 053612 (2001).

[29] S. Giorgini, "Collisionless dynamics of dilute Bose gases: Role of quantum and thermal fluctuations," Phys. Rev. A 61, 63615 (2000).

[30] B. Jackson and E. Zaremba, "Quadrupole collective modes in trapped finite-temperature Bose-Einstein condensates," Phys. Rev. Lett. 88, 180402 (2002).

[31] S. Bhongale, R. Walser, and M. Holland, "Memory effects and conservation laws in the quantum kinetic evolution of a dilute Bose,” Phys. Rev. A 66, 043618 (2002).

[32] M. Naraschewski and R. J. Glauber, "Spatial coherence and density correlations of trapped Bose gases," Phys. Rev. A 59, 4595 (1999).

[33] I. Bloch, T. Hänsch, and T. Esslinger, "Measurement of the spatial coherence of a trapped Bose gas at the phase transition," Nature 403, 166 (2000).

[34] C. Orzel, A. Tuchman, M. Fenselau, and M. K. M. Yasuda, "Squeezed States in a Bose-Einstein Condensate," Science 291, 2386 (2001).

[35] N. D. Mermin and H. Wagner, "Absence of Ferromagnetism or Antiferromagnetism in One- or Two-Dimensional Isotropic Heisenberg Models,” Phys. Rev. Lett. 17, 1133 (1966).

[36] P. C. Hohenberg, "Existence of Long-Range Order in One and Two Dimensions," Phys. Rev. 158, 383 (1967).

[37] D. Petrov, D. Gangardt, and G. Shlyapnikov, "Low-dimensional trapped gases," cond-mat/0409230 (2004), and references therein.

[38] J. Andersen, U. A. Khawaja, and H. Stoof, "Phase Fluctuations in Atomic Bose Gases," Phys. Rev. Lett. 88, 70407 (2002).

[39] U. A. Khawaja, J. Andersen, N. Proukakis, and H. Stoof, "Low dimensional Bose gases," Phys. Rev. A 66, 13615 (2002).
[40] D. Hellweg, L. Cacciapuoti, M. Kottke, T. Schulte, K. Sengstock, W. Ertmer, and J. J. Arlt, "Measurement of the Spatial Correlation Function of Phase Fluctuating Bose-Einstein Condensates,” Phys. Rev. Lett. 91, 010406 (2003).

[41] M. Olshanii, "Atomic Scattering in the Presence of an External Confinement and a Gas of Impenetrable Bosons," Phys. Rev. Lett. 81, 938 (1998).

[42] C. Menotti and S. Stringari, "Collective oscillations of a onedimensional trapped Bose-Einstein gas," Phys. Rev. A 66, 043610 (2002).

[43] T. Stöferle, H. Moritz, C. Schori, M. Köhl, and T. Esslinger, "Transition from a Strongly Interacting 1D Superfluid to a Mott Insulator," Phys. Rev. Lett. 92, 130403 (2004).

[44] A. J. Moerdijk and B. J. Verhaar, "Prospects for Bose-Einstein condensation in atomic 7Li and 23Na," Phys. Rev. Lett. 73, 518 (1994).

[45] N. R. Newbury, C. J. Myatt, and C. E. Wieman, "s-wave elastic collisions between cold ground-state 87Rb atoms," Phys. Rev. A 51, 2680 (1995).

[46] S. Morgan, M. Lee, and K. Burnett, "Off-shell T matrices in one, two, and three dimensions," Phys. Rev. A 65, 22706 (2002).

[47] K. Huang, Statistical Mechanics (John Wiley \& Sons, Inc., New York, 1965).

[48] P. Lepage, "How to renormalize the Schrödinger equation," xxx.lanl.gov nucl-th/9706029 (1997).

[49] in Exactly Solvable Problems in Condensed Matter and Relativistic Field Theory, Vol. 242 of Lecture Notes in Physics, H. Araki, ed., (Springer, 1985).

[50] M. Girardeau, J. Math. Phys. 1, 516 (1960).

[51] R. Richardson, "Exactly solvable Many-Boson Model," J. Math. Phys. 9, 1327 (1968).

[52] J. Dukelsky and P. Schuck, "Condensate fragmentation in a new exactly solvable model for confined bosons," Phys. Rev. Lett. 86, 4207 (2001).

[53] K. Schönhammer and V. Meden, "Fermion-boson transmutation and comparison of statistical ensembles in one dimension," Am. J. Phys. 64, 1168 (1996).

[54] S. Chapman and T. G. Cowling, The Mathematical Theory of Non-Uniform Gases (Cambridge University Press, Cambridge, 1970).

[55] P. Ring and P. Schuck, The Nuclear Many-Body Problem (Springer Verlag, N.Y., 1980).

[56] A. I. Akhiezer and S. V. Peletminskii, Methods of Statistical Physics (Pergamon Press Ltd., Oxford, England, 1981).

[57] A. Abrikosov, L. Gor'kov, and I. Dzyaloshinskii, Quantum field theoretical methods in statistical physics (Pergamon Press, Oxford, England, 1965).

[58] J. P. Blaizot and G. Ripka, Quantum Theory of Finite Systems (The MIT Press, Cambridge, Massachusetts, 1986).

[59] J. R. Schrieffer, Theory of Superconductivity (Perseus Books, Cambridge, Massachusetts, 1999).

[60] W. P. Schleich, Quantum optics in Phase Space (Wiley-VCH, Berlin, Germany, 2001).

[61] A. Griffin, "Conserving and gapless approximations for an inhomogeneous Bose gas at finite temperatures," Phys. Rev. B 53, 9341 (1996).

[62] M. Girardeau and R. Arnowitt, "Theory of Many-Boson Systems: Pair Theory,” Phys. Rev. 113, 755 (1959).

[63] Y. Castin and R. Dum, "Low-temperature Bose-Einstein condensates in time-dependent traps: Beyond the $U(1)$ symmetrybreaking approach,” Phys. Rev. A 57, 3008 (1998).

[64] C. W. Gardiner and P. Zoller, "Quantum kinetic theory V: Quantum kinetic master equation for mutual interaction of conden- 
sate and noncondensate," Phys. Rev. A 61, 033601 (2000), and references therein.

[65] L. Kadanoff and G. Baym, Quantum Statistical Mechanics, Frontiers in Physics (W. A Benjamin, Inc., New York, 1962).

[66] J. Kane and L. Kadanoff, "Green's Functions and Superfluid Hydrodynamics," J. of Math. Phys. 6, 1902 (1965).

[67] G. Golub and C. V. Loan, Matrix Computations (Johns Hopkins Unversity Press, Baltimore, 1996).

[68] S. Morgan, "Response of Bose-Einstein condensates to external perturbations at finite temperature," Phys. Rev. A 69, 023609 (2004).

[69] M. Lewenstein and L. You, "Quantum Phase Diffusion of a Bose-Einstein Condensate," Phys. Rev. Lett. 77, 3489 (1996).

[70] L. You, W. Houston, M. Lewenstein, and M. Marinescu, "Low energy excitations of a trapped Bose condensates," Act. Phys. Pol. A 93, 211 (1998).

[71] R. Walser, J. Cooper, and M. Holland, "Reversible and irreversible evolution of a condensed bosonic gas," Phys. Rev. A 63, 013607 (2001).
[72] A. Görlitz, J. Vogels, A. Leanhardt, C. Raman, T. G. ad J. AboShaeer, A. Chikkatur, S. Gupta, S. Inouye, T. Rosenband, and W. Ketterle, "Realization of Bose-Einstein Condensates in Lower Dimensions,” Phys. Rev. Lett. 87, 130402 (2001).

[73] H. Ott, J. Fortagh, G. Schlotterbeck, A. Grossmann, and C. Zimmermann, "Bose-Einstein Condensation in a Surface Microtrap,” Phys. Rev. Lett. 87, 230401 (2001).

[74] J. Dobson, "Harmonic-Potential Theorem: Implications for Approximate Many-Body Theories," Phys. Rev. Lett. 73, 2244 (1994).

[75] B. D. Esry and C. H. Greene, "Superfluids mixing it up," Nature 392, 434 (1998).

[76] R. H. Brown and R. Twiss, "Correlation between photons in two coherent beams of light," Nature 177, 27 (1956).

[77] R. Glauber, "The Quantum Theory of Optical Coherence," Phys. Rev. 130, 2529 (1963).

[78] R. Walser, "Quantenfelder à la carte," Physik Journal 11, 19 (2002). 